FERMILAB

DESY 01-056

July 2001
JUL 272001

LIBRARY

\title{
Study of Frequency Multiplication Process in Multistage HGHG FEL
}

W. Brefeld, B. Faatz, J. Feldhaus, M. Körfer, T. Möller,

J. Pflueger, J. Rossbach, E. L. Saldin, E. A. Schneidmiller, S. Schreiber Deutsches Elektronen-Synchrotron DESY, Hamburg

\section{J. Krzywinski}

Institute of Physics of the Polish Academy of Sciences, Warsaw, Poland

\section{V. Yurkov}

Joint Institute for Nuclear Research, Dubna, Moscow Region, Russia

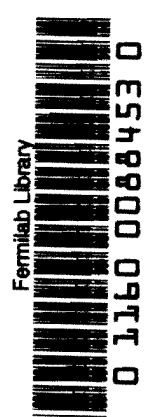

ISSN 0418-9833

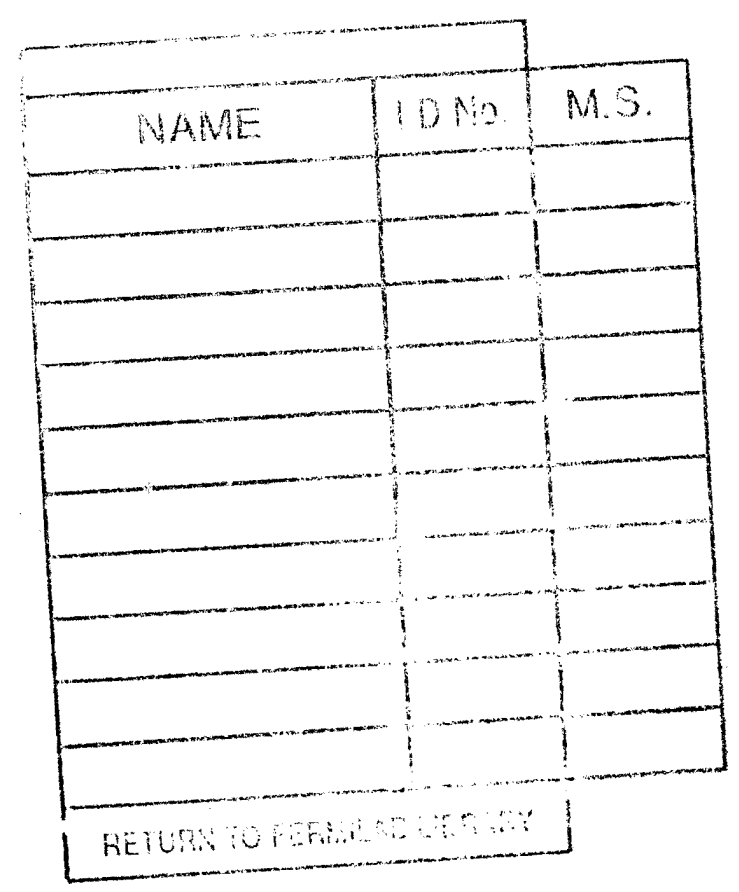




\title{
Study of Frequency Multiplication Process in Multistage HGHG FEL
}

\author{
W. Brefeld ${ }^{\text {a }}$, B. Faatz ${ }^{\text {a }}$, J. Feldhaus ${ }^{\text {a }}$, M. Körfer ${ }^{\text {a }}$, \\ J. Krzywinski ${ }^{\text {b }, ~ T . ~ M o ̈ l l e r ~}{ }^{\text {a }}$, J. Pflueger ${ }^{\text {a }}$, J. Rossbach ${ }^{\text {a }}$, \\ E.L. Saldin ${ }^{\text {a }}$, E.A. Schneidmiller ${ }^{\text {a }}$ S. Schreiber ${ }^{\mathrm{a}}$ and \\ M.V. Yurkov ${ }^{c}$ \\ a Deutsches Elektronen-Synchrotron (DESY), Notkestrasse 85, D-22607 Hamburg, \\ Germany \\ ${ }^{\mathrm{b}}$ Institute of Physics of the Polish Academy of Sciences, 02688 Warszawa, Poland \\ ${ }^{\mathrm{c}}$ Joint Institute for Nuclear Research, Dubna, 141980 Moscow Region, Russia
}

\begin{abstract}
A new design for multistage High-Gain Harmonic Generation (HGHG) scheme is proposed. Each stage (except first) consists of radiator undulator, dispersion section (demodulator), FEL amplifier and end-stage dispersion section (modulator). The second harmonic in the $n$th stage becomes the fundamental in the $(n+1)$ th stage. The main difference with previous HGHG schemes is that in our scheme the HGHG technique can be applied more than once in a HGHG chain (single bunch scheme). This is possible because the density modulation exiting each stage is relatively small, $10 \%$ and $1 \%$ for the fundamental and the second harmonic, respectively. Hence, a small energy modulation is sufficient to create this microbunching in the dispersion section. In this case the growth of the energy spread due to the HGHG process is much less than initial energy spread, and exponential growth rate in the main undulator is practically the same as without stage sequence. At chosen parameters for each stage the amplitude of second harmonic of density modulation dominates significantly over the amplitude of shot noise harmonic, and the modulation of the beam density can be used as input signal for the next HGHG stage. Problems relating to X-ray HGHG FEL are discussed. Our studies have shown that the frequency multiplication process produces a noise degradation proportional to at least the square of the multiplication ratio. This prevents operation of HGHG FEL at a very short wavelength range. The results presented in this paper have demonstrated that the HGHG FEL approach is quite adequate for the VUV coherent source, but not scalable to X-ray devices.
\end{abstract}




\section{Introduction}

Recent advances in accelerator techniques reveal the perspective to construct in the nearest future free electron lasers capable to operate in the X-ray wavelength band. Several projects of such FELs are now under development $[1,2]$. The common feature of these projects is that they are based on a single pass SASE FEL scheme. Peak and average brilliance of these sources of coherent radiation exceed significantly the corresponding values presently available at the most advanced storage ring synchrotron radiation sources. However, the quality of the output radiation of the single pass SASE FEL is still far from ideal because of its short longitudinal coherence length. This is a consequence of the fact that the process of amplification in the SASE FEL starts from shot noise. As a result, the output radiation consists of a number of independent wavepackets (or spikes). The length of each wavepacket is much less than the radiation pulse length and there is no phase correlation between the wavepackets. Therefore, the improvement of the longitudinal coherence of the X-ray SASE FEL is of great practical importance.

At present two ways to overcome this problem are under development. One of them is based on an idea to use a self-seeding scheme [3-5]. In this scheme the FEL consists of two undulators and a monochromator located between them. The first undulator operates in the linear regime of amplification starting from shot noise, and the output radiation has usual SASE properties. After the exit of the first undulator the electron beam is guided through a bypass, and the radiation enters the monochromator which selects a narrow band of radiation. At the entrance of the second undulator the monochromatic radiation is combined with the electron beam and is amplified up to the saturation level. The output radiation of this two-stage FEL possesses all the features which usually refer to laser radiation: full transverse and longitudinal coherence of the radiation within the radiation pulse, and stability of the output power [3,4].

Another approach to produce completely coherent radiation consists in utilizing a highgain harmonic generation (HGHG) FEL scheme. In the HGHG FEL the radiation output is derived from a coherent subharmonic seed pulse. Consequently, the optical properties of the HGHG FEL are expected to be a map of the characteristics of the high-quality seed laser. This has the benefit of providing radiation with a high degree of stability and control of the central wavelength, bandwidth energy and pulse duration which is absent from the SASE FEL source [6-10]. An idea of using two undulators, with the second undulator resonant to one of the harmonics of the first one, has been proposed in [6] to generate UV radiation. The first undulator is long enough to reach saturation and produce strong spatial bunching in harmonics. The bunched beam generates coherent radiation in the second undulator which follows immediately the first one. The next step in this direction was taken in [7], where the following modifications of the scheme have been introduced:

(1) The first undulator is shortened significantly. The input laser power is controlled in such a way that the maximum energy modulation of the electron beam is about equal to the energy spread, but still far away from saturation.

(2) A dispersion section is introduced between the two undulators and optimized to generate maximum harmonics in the spatial bunching.

(3) The second undulator is long enough in order to provide exponential growth of the 

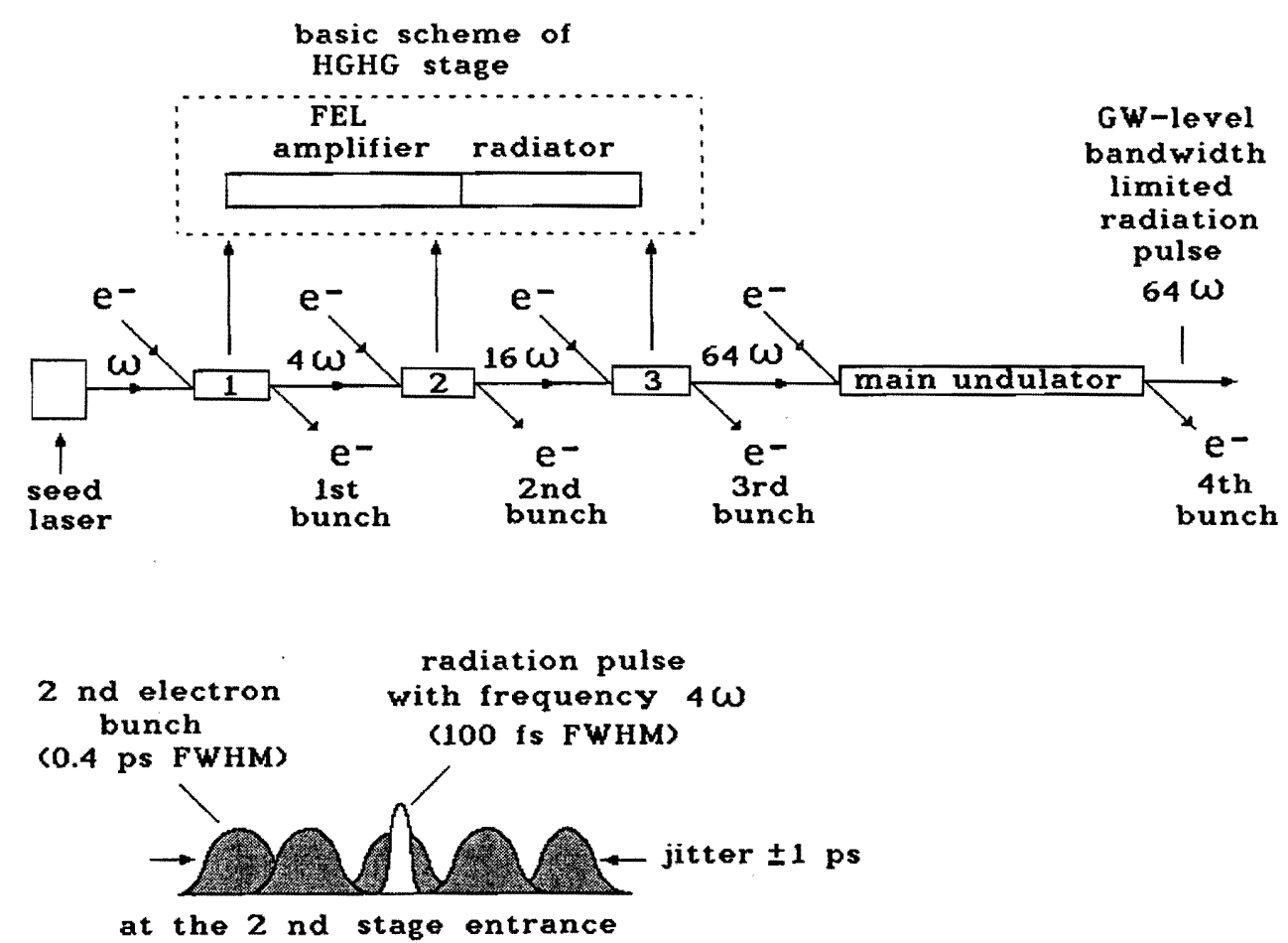

Fig. 1. Up: HGHG multistage scheme proposed in [13]. Down: Schematic depiction of the time structure of seed radiation pulses showing their synchronization with electron pulses at the stage entrances

radiation power. This becomes possible because of the proper control of the energy modulation in the first undulator.

Recently, an approach utilizing a HGHG scheme, which is capable of producing longitudinally coherent pulses, was demonstrated experimentally $[11,12]$. A seed laser at the wavelength of $10.6-\mu \mathrm{m}$ was used to generate amplified FEL output at $5.3-\mu \mathrm{m}$. Measurements of frequency spectrum, pulse duration and correlation length of the 5.3- $\mu \mathrm{m}$ output radiation verified that the light is fully longitudinally coherent.

To generate short wavelengths we have to go to a high harmonic number. In order to obtain an efficient coherent harmonic generation, the energy modulation $\delta E$, introduced by the modulator, needs to be larger than the initial energy spread by the factor of harmonic number $N$. Therefore, for very high harmonics, the energy modulation becomes very large and this makes the exponential growth gain length too large. This problem can be solved with a multistage HGHG scheme [13] (see Fig. 1). The technique of using an FEL amplifier as a frequency multiplier relies on the fact that spatial bunching in the exponential gain regime can be very strong. Close to saturation, quite strong bunching and a large amplitude of harmonic radiation are generated. At the end of exponential linear regime (closer to saturation) it is possible to change the undulator parameters abruptly in order to bring the higher harmonic radiation into resonance with the undulator. Because the beam has a large component of bunching at a short wavelength, coherent emission is copiously produced. The main problem with this approach is the large energy spread which will be generated by the bunching of the electron beam at the fundamental frequency. While this energy spread is necessary for the bunching, it degrades the per- 


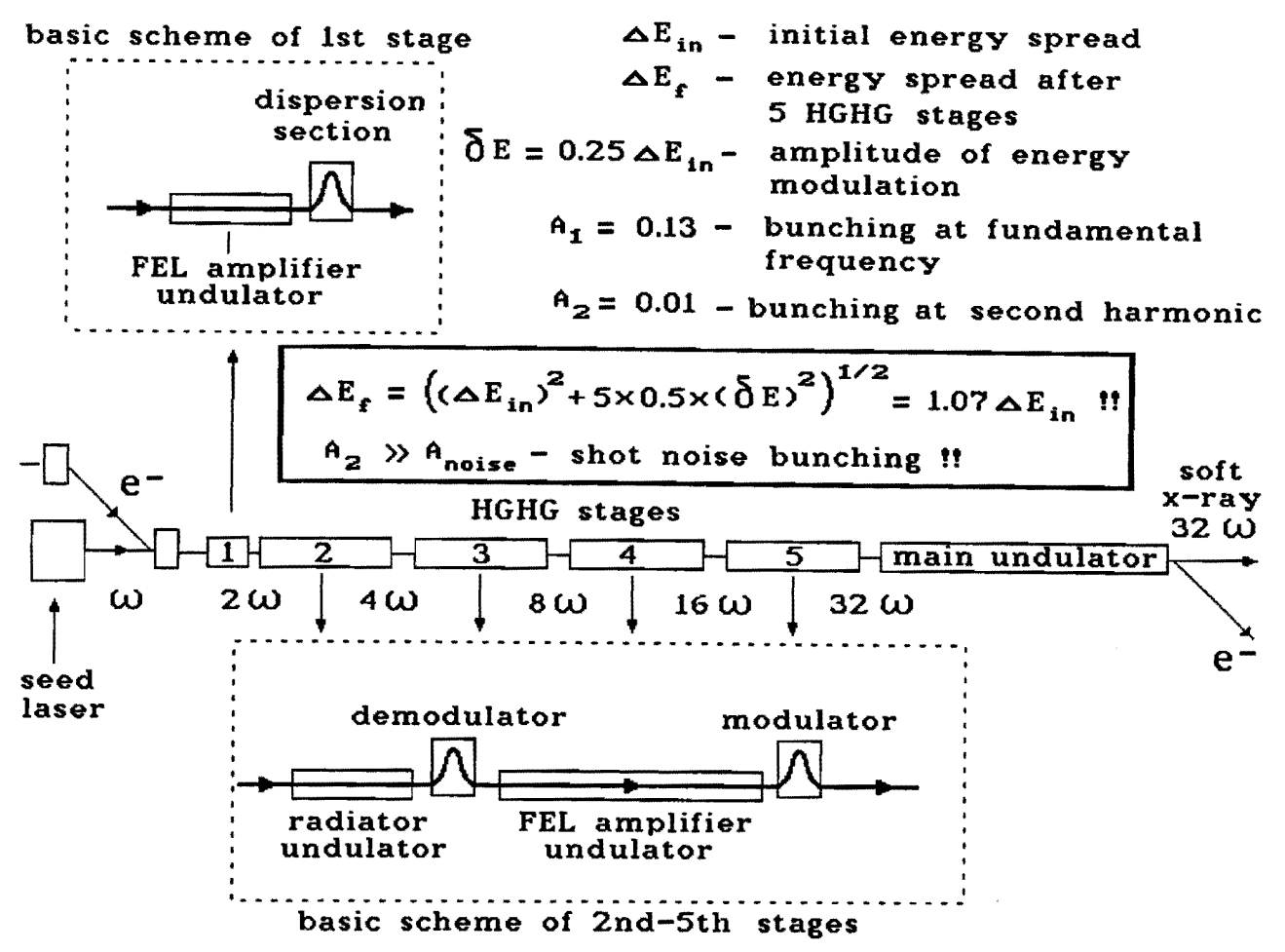

Fig. 2. Single bunch HGHG FEL scheme proposed in this paper

formance of the FEL amplifier section at the harmonic frequency. A simple solution to solve the energy spread problem, which has been proposed in [13], is to use fresh electron bunches in each stage. Once the coherent harmonic generation process in the first stage is over, the resulting radiation at frequency $4 \omega$ is guided to the next amplifier/radiator unit for interaction with a fresh electron bunch. (see Fig. 1). Following the second stage the radiation at frequency $16 \omega$ enters the third stage. Like the second stage, the third stage makes use of fresh electron bunches, etc. The results of these studies are considered very promising because they indicate that the HGHG FEL technique could allow the production of fully coherent X-rays [13].

In this paper we propose an idea for a single-bunch, multistage HGHG FEL scheme (see Fig, 2). Each stage (except the first stage) consists of a radiator undulator, first dispersion section (demodulator), FEL amplifier undulator and end-stage dispersion section (modulator). The proposed scheme operates as follows. The first stage is a conventional FEL amplifier seeded by an external laser. The seed laser pulse is timed to overlap with the electron bunch. This laser pulse interacts with the electron beam in the first undulator, which is tuned to be resonant to the seed radiation wavelength. The undulator is followed by a dispersion section to increase spatial bunching. In contrast to usual the HGHG approach [7], in our scheme the density modulation at the fundamental frequency at the exit of the dispersion section is about $10 \%$ only. Nevertheless, at the chosen parameters, the amplitude of the second harmonic of the density modulation is high enough, about $1 \%$, and dominates significantly over the amplitude of shot noise harmonics (about $0.01 \%$ ). This modulation density serves as an input signal for the second stage which is resonant to the second harmonic. An important feature of our design is that a very small energy modulation is sufficient to produce $10 \%$ microbunching in the dispersion section. 
In particular, the amplitude of the energy modulation $\delta E$ can be much smaller than the natural (local) energy spread in the electron beam $\Delta E_{\text {in }}$. The analysis of the parameters of the subharmonically seeded FEL has shown that it will operate reliably even for an energy modulation amplitude equal to $\delta E=0.25 \Delta E_{\text {in }}$ (see Sec.2 for more details).

Figure 2 illustrates how the soft X-ray wavelength range may be reached by successive multiplication $(\omega \rightarrow 2 \omega \rightarrow 4 \omega \rightarrow 8 \omega \rightarrow 16 \omega \rightarrow 32 \omega)$ in a stage sequence. Following the first stage (FEL amplifier undulator and dispersion section) the electron beam and the seed radiation enter a short undulator (radiator) which is resonant with the second harmonic of the seed radiation. In the radiator the seed radiation plays no role and is diffracted out of the electron beam, while the bunched beam generates radiation with frequency $2 \omega$. At the exit of the radiator undulator the radiation power exceeds significantly the effective power of shot noise. After leaving the radiator the electron beam is guided through a dispersion section (demodulator). The function of this dispersion section consists in suppressing the density and energy modulation of the electron bunch produced in the first stage. The problem of suppressing the beam modulation can be solved quite naturally due to the presence of the local energy spread in the electron beam. After passing the dispersion section, the demodulated electron beam enters the FEL amplifier and amplifies in exponential regime the radiation with frequency $2 \omega$ produced by the radiator undulator. The length of the FEL amplifier undulator is chosen in such a way that the energy modulation at the undulator exit has the same value of $\delta E=0.25 \Delta E_{\text {in }}$ as at the exit of the first stage. This energy modulation is then converted to spatial bunching while the electron beam traverses the end-stage dispersion section (modulator). The values of the 2nd and the 4th harmonics of density modulation at the second stage exit are about $10 \%$ and $1 \%$, respectively. These values are approximately the same as the amplitudes of the 1 st and 2nd harmonics at first stage exit. Following the second stage the beam enters the third stage which is resonant with 4 th harmonic of the seed radiation, etc. Finally, after the 5 th stage the electron beam enters main undulator which is resonant to the 32 th harmonic of the seed radiation. The process of amplification in the main undulator starts from the modulation of the beam density. By the time the beam is overbunched in the main undulator, the $32 \omega$ radiation reaches saturation. In order to reach saturation, the main undulator should be sufficient long. An important feature of the proposed scheme is that the energy modulation (i.e. correlated energy spread) induced in the $n$th stage, transforms to local (i.e. uncorrelated) energy spread in the $(n+1)$ th stage. As a result, the dispersion of the electron energy distribution at the exit of the multistage scheme is calculated as the sum of induced dispersions. The small energy perturbation of the electron beam is one of the advantages of the adopted subharmonically seeded FEL design. For instance, the total energy spread generated to the end of the 5th stage, can be estimated as $\sqrt{\left\langle(\Delta E)^{2}\right\rangle} \simeq \sqrt{\left(\Delta E_{\text {in }}\right)^{2}+5 \times 0.5 \times(\delta E)^{2}} \simeq 1.07 \Delta E_{\text {in }}$ for $\delta E=0.25 \Delta E_{\text {in }}$ which does not differ much from the number obtained from numerical simulations (see Sec. 5 for more details). Such a small degradation of the energy spread allows effective generation of powerful radiation in the main undulator.

Let us compare the problems of technical realization of the multistage HGHG scheme proposed in [13] (see Fig.1) and that proposed in our paper (see Fig.2). Clearly, the unique aspect of the first multistage scheme is its multi-bunch mode of operation. Typically, the electron bunch length for the soft X-ray FEL is of about $200 \mathrm{fs}$ (FWHM). The synchro- 
nization of the radiation pulse with the fresh electron bunch to within $200 \mathrm{fs}$ at each stage entrance is the most challenging task of first proposal. The main problem is the time jitter $( \pm 1 \mathrm{ps})$ of the electron and radiation pulses. The jitter of the electron pulses originates in the accelerator photoinjector and in the magnetic bunch compressors (from predicted $\pm 0.1 \%$ electron energy jitter). Due to this uncertainty not every radiation pulse will produce high harmonic radiation pulse at the HGHG FEL exit. The predicted probability of positioning the interaction region on the electron bunch is about $10 \%$ only for one stage. For three stages probability will decrease down to $0.1 \%$. Another problem of multi-bunch scheme [13] shown in Fig. 1 is that of the production of a sequence of electron bunches. One of the solutions could be the construction of separate driving accelerators for each stage. However, the accelerator costs are prohibitive (especially those capable to produce high peak current, high quality electron bunches). Another possible solution could be the construction of an accelerator capable to generate sequences of closely spaced electron bunches. Here another severe obstacle arises. Bunch separation can not be less than rf wavelength $(10 \mathrm{~cm}$ for S-band, and $24 \mathrm{~cm}$ for L-band). This means that special delay lines (bypasses) must be installed between HGHG stages. The bypass system needed for HGHG scheme has to satisfy the following requirements. First, the extra path length of the electron bunch going through the bypass must be equal to the bunch separation in the train which can not be less than 10 or $24 \mathrm{~cm}$. Second, passing the electron bunch through the bypass must avoid the emittance dilution due to coherent synchrotron radiation (CSR) effects. For very short bunches, CSR can generated energy spread in the bypass dipoles and thus dilute the horizontal emittance. For example, the bypass designed for soft X-ray SASE FEL at TTF at DESY has extra path length $1.2 \mathrm{~cm}$ only, but its length is equal to 21 meters, the bypass dipole bending angle is about half degree only. The dilution of the emittance in this bypass is about $10 \%$. Comparing the extra pass length of the TTF bypass $(1.2 \mathrm{~cm})$ with the $\mathrm{rf}$ wavelength of the accelerator $(10$ or $24 \mathrm{~cm})$, we can conclude that the length of such a HGHG stage delay line, about one hundred meters, is considered to be prohibitive.

The situation is quite different for multistage HGHG scheme proposed in our paper. Since it is a single bunch scheme, it does not require many accelerators or special time delay lines. In our case each stage produces higher harmonic radiation pulses which are strictly synchronized with the electron bunch at the entrance of the next stage. Since the input radiation pulse at the entrance of each stage is produced by the same electron bunch, the proposed scheme tolerates electron pulse jitter of about one picosecond.

We also performed a detailed study of the process of amplification in the HGHG FEL schemes. Up to now operation of HGHG schemes has been analyzed within the framework of idealized models which do not take into account shot noise effects [7-10]. The results of these studies were very promising and allowed the authors to make the conclusion that HGHG FEL technique would allow to reach the X-ray wavelength range starting from visible light. In this paper we take into account shot noise in the electron beam. It has been found that a general disadvantage of HGHG FEL schemes (as well as any frequency multiplication scheme) is due to strong noise degradation of the properties of output radiation with increasing harmonic number $N$. In the case of HGHG FEL this means that the effect of frequency multiplication by a factor of $N$ results in multiplication of the ratio of noise power to carrier by a factor of $N^{2}$. This prevents successful operation of 
HGHG FEL at very short wavelengths. On the basis of our study we can make the definite conclusion that the applicability region of the HGHG scheme is significantly narrower than claimed before. The results presented in this paper demonstrate that the HGHG scheme is quite adequate for the 10-100 $\mathrm{nm}$ coherent light source, but cannot be used to produce hard X-rays. It is explicitly shown that noise degradation prevents operation HGHG multistage scheme at Angstrom wavelength range.

The paper proceeds as follows. In Sec. 2 we describe the basic ideas of the singlebunch HGHG scheme. Section 3 is devoted to the analysis of space charge and coherent synchrotron radiation effects in the modulated electron beam moving along the HGHG chain. Section 4 discusses the noise properties of HGHG FEL. In section 5 we present specific numerical example of multistage HGHG FEL. The parameter optimization and performance characterizations are performed with 3-D time-dependent simulation code. In section 6 we analyze the problem of an X-ray HGHG FEL.

\section{General description of subharmonically seeded multistage HGHG FEL}

The layout of the subharmonically seeded FEL is shown schematically in Fig. 3. Facility for production of fully coherent soft X-ray pulses consists of a seed laser, five stages of frequency doubling and main undulator. The first stage of frequency doubling $(260 \rightarrow$ $130 \mathrm{~nm}$ ) consists of FEL amplifier undulator, and dispersion section for the beam density modulation. The scheme operates as follows. The seed laser pulse of 5 ps duration and 10 MW peak power is timed to overlap with the electron bunch. This laser pulse interacts with the electron beam in the first undulator, which is tuned to be resonant to seed radiation wavelength. The top of the seed radiation pulse of 5 ps duration can be easily tuned to the arrival time of the short electron bunch at the predicted absolute jitter of about \pm 1 ps. Radiation power is exponentially amplified upon passing through the first undulator. The amplitude of energy modulation of the electron beam at the undulator exit is equal to $0.3 \mathrm{MeV}$, while the beam density modulation is not sufficient to drive the second stage. The required value of the beam bunching at the second harmonic of about 0.01 is achieved when the electron beam passes through dispersion section (the density modulation at the fundamental frequency is about 0.1 in this case). In this case the amplitude of the second harmonic of the density modulation dominates significantly over the amplitude of shot noise harmonic (of about $0.01 \%$ ), and it serves as input signal for the second stage.

Optimal parameters of the dispersion section can be calculated in the following way. The phase space distribution of the particles in the first FEL amplifier is described in terms of the distribution function $f(P, \psi)$ written in "energy-phase" variables $P=E-E_{0}$ and $\psi=k_{\mathrm{w}} z+\omega_{0}(z / c-t)$, where $E_{0}$ is the nominal energy of the particle, $k_{\mathrm{w}}=2 \pi / \lambda_{\mathrm{w}}$ is the undulator wavenumber, and $\omega_{0}$ is the frequency of the seed radiation. Before entering the first undulator, the electron distribution is assumed to be Gaussian in energy and uniform in phase $\psi$ :

$$
f_{0}(P, \psi)=\frac{1}{\sqrt{2 \pi\left\langle(\Delta E)^{2}\right\rangle}} \exp \left(-\frac{P^{2}}{2\left\langle(\Delta E)^{2}\right\rangle}\right)
$$




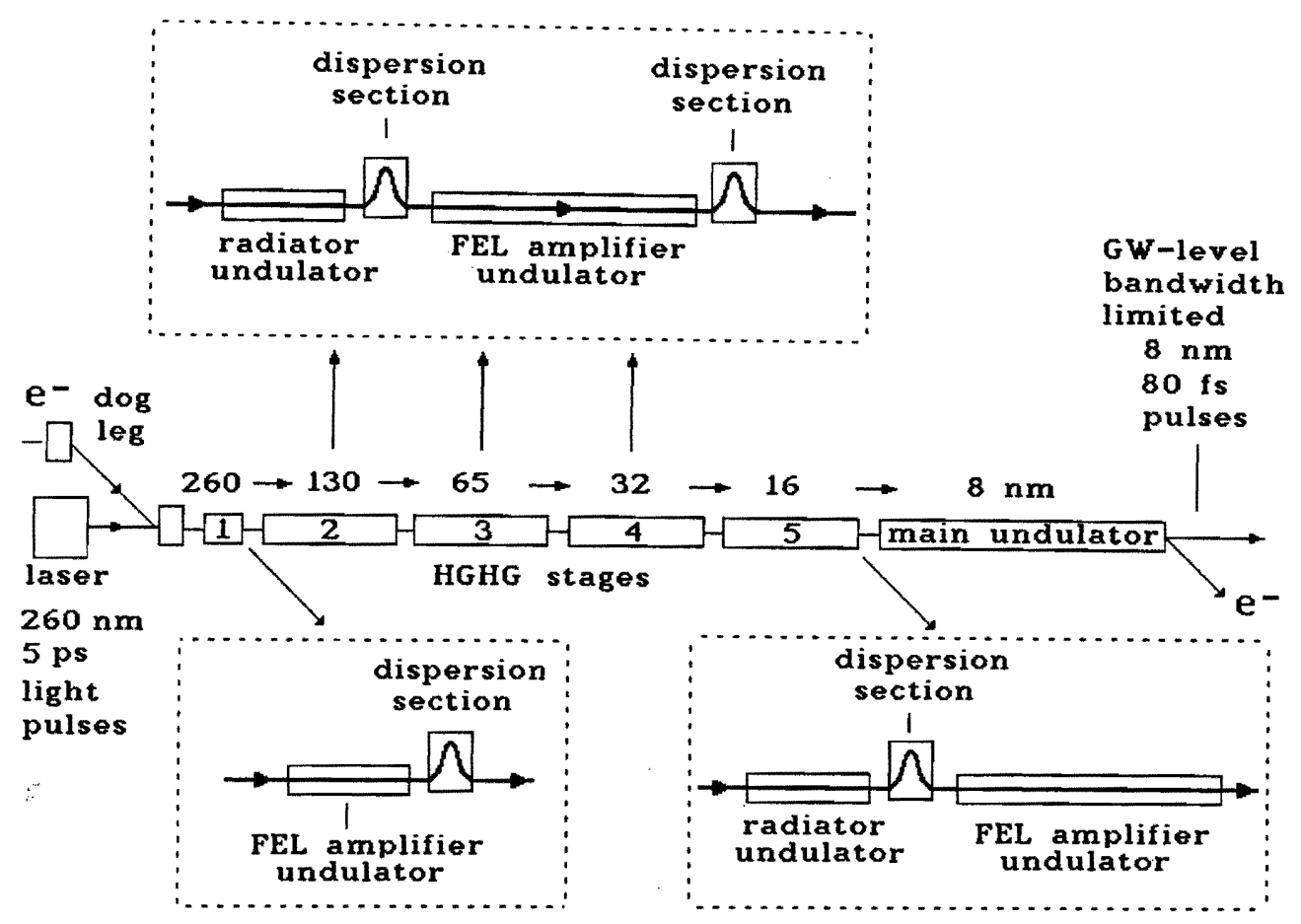

Fig. 3. Schematic layout of subharmonically seeded soft X-ray FEL at TTF at DESY

The present study assumes the density modulation at the end of first undulator to be very small compared to the desired value $(10 \%)$ and there is an energy modulation $P_{0} \sin \psi$ only. Then the distribution function at the entrance to the dispersion section is

$$
f_{0}\left(P+P_{0} \sin \psi\right) \text {. }
$$

After passing through the dispersion section with dispersion strength $\mathrm{d} \psi / \mathrm{d} P$, the electrons of phase $\psi$ and energy deviation $P$ will come to a new phase $\psi+P \mathrm{~d} \psi / \mathrm{d} P$. Hence, the distribution function becomes

$$
f(P, \psi)=f_{0}\left(P+P_{0} \sin \left(\psi-P \frac{\mathrm{d} \psi}{\mathrm{d} P}\right)\right) .
$$

The integration of this distribution over energy provides the beam density distribution, and the Fourier expansion of this function gives the harmonic components of the density modulation converted from the energy modulation [7]:

$$
\begin{aligned}
& \int_{-\infty}^{\infty} f(P, \psi) \mathrm{d} P=1+2 \sum_{n=1}^{\infty} \exp \left[-\frac{1}{2} n^{2}\left\langle(\Delta E)^{2}\right\rangle\left(\frac{\mathrm{d} \psi}{\mathrm{d} P}\right)^{2}\right] \\
& \times J_{n}\left(n P_{0} \frac{\mathrm{d} \psi}{\mathrm{d} P}\right) \cos (n \psi) .
\end{aligned}
$$

The Bessel function factor represents the microbunching. If its argument is much smaller than unity, the microbunching would be reduced proportionally to its $n$th power. Hence, 
$P_{0} \mathrm{~d} \psi / \mathrm{d} P$ must be comparable to $a_{1}$, where $a_{1}$ is the desirable value of first harmonic bunching factor. The first exponential factor shows that the energy spread suppresses significantly the microbunching when $\Delta E \mathrm{~d} \psi / \mathrm{d} P \simeq a_{1} \sqrt{\left\langle(\Delta E)^{2}\right\rangle} / P_{0}$ is larger or equal to unity. Hence, the energy modulation can be smaller than the energy spread in order to have a small first harmonic components $\left(a_{1} \ll 1\right)$. Parameters in our case are: $\sqrt{\left\langle(\Delta E)^{2}\right\rangle} \simeq 1$ $\mathrm{MeV}, P_{0} \simeq 0.25 \mathrm{MeV}, P_{0} \mathrm{~d} \psi / \mathrm{d} P \simeq 0.16$. For these parameters we find the amplitudes of the first and the second harmonic of density modulation:

$$
\begin{aligned}
& 2 J_{1}\left(P_{0} \frac{\mathrm{d} \psi}{\mathrm{d} P}\right) \exp \left[-\frac{1}{2}\left\langle(\Delta E)^{2}\right\rangle\left(\frac{\mathrm{d} \psi}{\mathrm{d} P}\right)^{2}\right] \simeq 0.13, \\
& 2 J_{2}\left(2 P_{0} \frac{\mathrm{d} \psi}{\mathrm{d} P}\right) \exp \left[-2\left\langle(\Delta E)^{2}\right\rangle\left(\frac{\mathrm{d} \psi}{\mathrm{d} P}\right)^{2}\right] \simeq 0.01 .
\end{aligned}
$$

The above consideration of the bunching process has been treated within the framework of a single particle dynamic theory. One should care also about avoiding coherent synchrotron radiation (CSR) effects. The electric field of CSR induces an energy modulation along the bunch. Calculations of the correlated energy spread growth due to CSR shows that this should not be a limitation in our case (see Sec. 3 for more details).

Another harmful effect which may influence the operation of a HGHG scheme is the space charge. Calculations of the space charge force in the beam bunched at $260 \mathrm{~nm}$ show that this should be a serious limitation in our case. The electric field of the space charge induces the additional energy modulation at $260 \mathrm{~nm}$ wavelength. If we calculate plasma oscillation along the HGHG scheme, the space charge induced energy modulation is predicted to be about $0.5 \mathrm{MeV}$. Specific design of the second stage is proposed for preparation of the electron beam with the required energy spread in order to overcome the problem of plasma oscillation. The design configuration of the second stage is shown in Fig. 4. The stage consists of a short undulator (radiator), dispersion section (demodulator), high-gain FEL amplifier and end-stage dispersion section (modulator).

Following the first stage the beam and seed radiation enter short undulator (radiator) which is resonant with second harmonic of seed radiation $(130 \mathrm{~nm})$. In the radiator the seed radiation plays no role and is diffracted out of the electron beam. However, a new 130 $\mathrm{nm}$ radiation is generated by the density-modulated electron beam and rapidly reaches MW-level peak power. After the radiator the electron beam is guided through a dispersion section ( magnetic chicane) and the $130 \mathrm{~nm}$ radiation beam enters the grazing mirror system (see Fig. 4). The function of this chicane consists in suppressing the $260 \mathrm{~nm}$ modulation of the electron bunch produced in the first stage. The function of the mirror system consists in making the path length of the electron and the radiation beam to be equal. The trajectory of the electron beam in the chicane has the shape of an isosceles triangle with the base equal to $L$. The angle adjacent to the base, $\theta$, is considered to be small. The problem of suppressing the beam modulation induced in the first stage can be solved quite naturally due to the presence of the local energy spread in the electron beam. Parameters in our case are: $\theta=0.6^{\circ}, L=3 \mathrm{~m}$, compaction factor $R_{56}=L \theta^{2} \simeq 300 \mu \mathrm{m}$, $\sqrt{\left\langle(\Delta E)^{2}\right\rangle} R_{56} / E_{0} \simeq 0.3 \mu \mathrm{m}, \lambda=0.26 \mu \mathrm{m}$. This leads to the suppression of the electron 


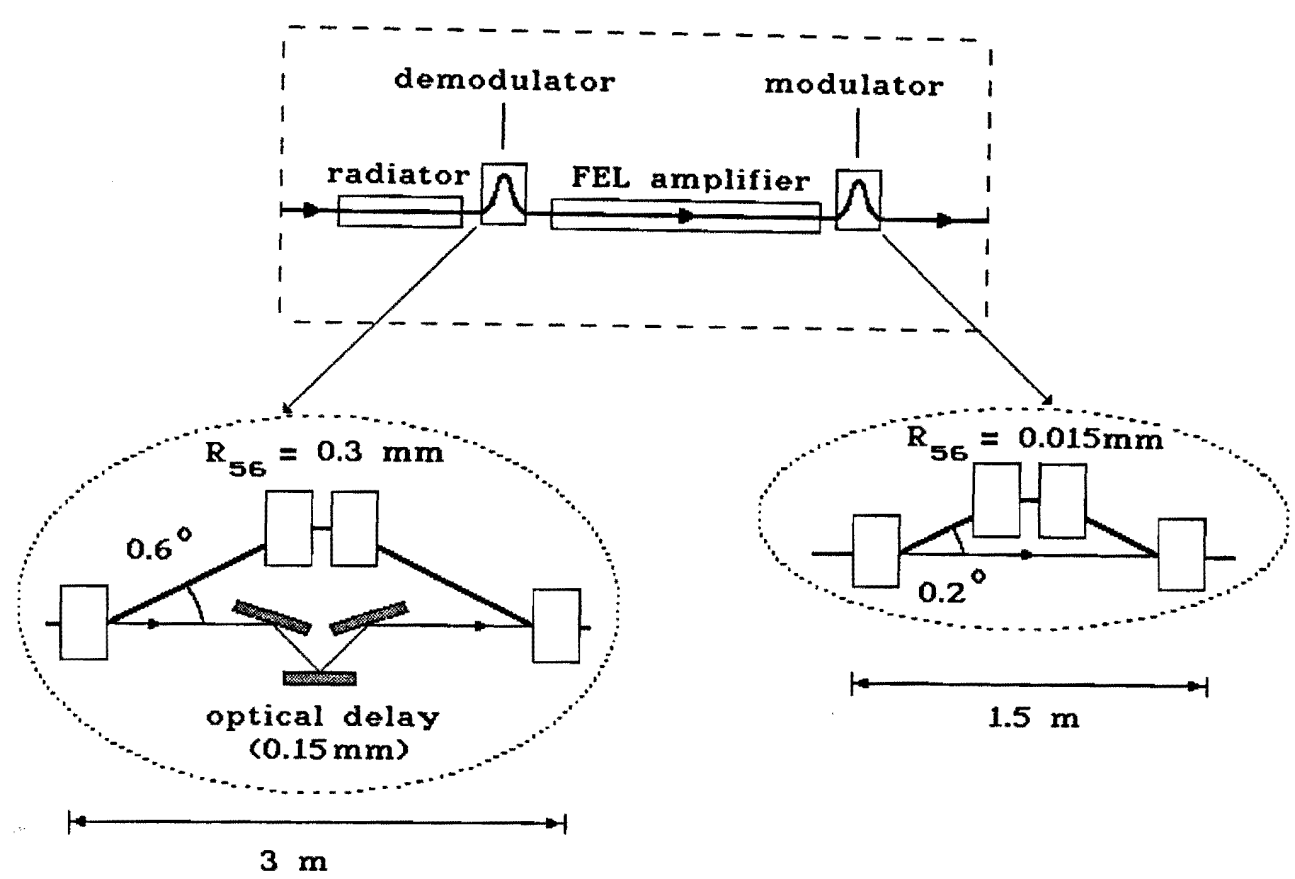

Fig. 4. Schematic illustration of design configuration for the second stage of subharmonically seeded soft X-ray FEL at TTF

beam modulation by a factor $\exp (-20)$ (for Gaussian energy distribution).

Passing the chicane the demodulated electron beam and seed $130 \mathrm{~nm}$ radiation enter the FEL amplifier undulator. Upon passing through the FEL amplifier radiation is exponentially amplified. This undulator is long enough to reach $0.25 \mathrm{MeV}$ energy modulation at the wavelength of $130 \mathrm{~nm}$. Since the density modulation at $130 \mathrm{~nm}$ is of about 4 times smaller than required value $(0.2)$, one should use dispersion section at the exit of the second stage. After passing second dispersion section, the energy modulation induced in the beam by amplification in the FEL amplifier transforms into the density modulation.

Following the second stage the beam enters the third stage. Like the second stage, the third stage also consists of the radiator (which is resonant to the 4th harmonic of the seed radiation), demodulator, FEL amplifier and modulator. Now $65 \mathrm{~nm}$ density modulation serves as a seed for this radiator, etc. Main undulator, resonant with 32th harmonic of seed radiation $(8 \mathrm{~nm})$, follows immediately after the 5 th stage. By the time the beam is overbunched in the main undulator, the $8 \mathrm{~nm}$ radiation reaches $\mathrm{GW}$ level. In order to reach saturation, the main undulator should be sufficiently long. Calculations of the space charge effects show that they are negligible in the main undulator. The electric field of space charge is proportional to the wavelength of density modulation $\lambda$. As a result, the amplitude of energy modulation at $16 \mathrm{~nm}$ is much less than energy spread, and there is no need to install special chicane for suppressing the beam density modulation induced in the 5 th stage. Tables 1, 2, and 3 list some of the basic parameters of the electron beam, seed laser system, and magnetic system of HGHG FEL.

In our proposal soft X-ray pulses are produced by the multistage HGHG technique. Frequency multiplication can be an essential pulse shortening mechanism. Because of the nonlinearity of the conversion process, one expects the generation of double frequency 
Table 1

Parameters of the electron beam at the TESLA Test Facility accelerator

\begin{tabular}{|ll|}
\hline bunch energy, $\mathrm{MeV}$ & $500-1000$ \\
bunch charge, $\mathrm{nC}$ & 1 \\
rms bunch length, $\mu \mathrm{m}$ & 50 \\
rms energy spread, $\%$ & 0.1 \\
normalized emittance, $\pi$ mm-mrad & 2 \\
number of bunches per train & 7200 \\
bunch spacing, $\mathrm{ns}$ & 111 \\
repetition rate, $\mathrm{Hz}$ & 10 \\
\hline
\end{tabular}

Table 2

Parameters of the seed laser

\begin{tabular}{|ll|}
\hline wavelength, nm & $260-800$ \\
pulse duration, ps (FWHM) & 5 \\
energy per pulse, $\mu \mathrm{J}$ & 50 \\
spectrum width & transform limited \\
number of bunches per train & 7200 \\
bunch spacing, ns & 111 \\
repetition rate, $\mathrm{Hz}$ & 10 \\
time jitter, ps & 1 \\
\hline
\end{tabular}

pulse $\sqrt{2}$ times shorter than the pulse duration at the fundamental frequency (for Gaussian axial profile). Successive multiplication to the $N$ th harmonic resulting in $\sqrt{N}$-fold compression of the $N$ th harmonic pulse duration. In our case $N=32$, and numerical simulations show that we can obtain 80 fs pulse at the wavelength of $8 \mathrm{~nm}$.

Let us discuss the problem for production ultrashort pulses. HGHG scheme can be combined with a femtosecond optical quantum laser (see Fig. 5). An ultrashort laser pulse is used to seed the first stage, and then the process of frequency multiplication is developed as it was described above. The synchronization of the femtosecond optical laser with the electron pulses to within $200 \mathrm{fs}$ is the most challenging task in this case. Femtosecond pulses from the laser system should be synchronized to the photoinjector master clock with phase-locking technique. The main problem is the time jitter $( \pm 1 \mathrm{ps})$ of electron and seed laser pulses. The jitter of electron pulses originates in the photoinjector laser systems (laser pulse jitter) and in magnetic bunch compressors (from predicted $\pm 1 \%$ electron bunch energy jitter). This means that we cannot use each femtosecond optical pulse to produce femtosecond X-ray pulse. The predicted probability of positioning the interaction region on the electron bunch is about $10 \%$ only, as can be verified easily from the sketch in Fig. 5.

Pump probe techniques which are commonly used with optical lasers, are highly desirable in order to make full use of the femtosecond soft X-ray pulses. Since, in this case, precise timing is needed with a jitter of less than $30 \mathrm{fs}$, we suggest to combine the femtosecond soft X-ray pulses with optical pulses generated in the seed laser system. An attractive feature of such an approach is that femtosecond X-ray pulses become to be naturally synchronized with the femtosecond optical pulses, thus cancelling jitter. 
Table 3

Parameters of the magnetic system of HGHG FEL scheme

\begin{tabular}{|c|c|c|c|c|c|c|}
\hline & $\begin{array}{l}\text { Stage 1 } \\
256 \mathrm{~nm} \\
\end{array}$ & $\begin{array}{l}\text { Stage } 2 \\
128 \mathrm{~nm} \\
\end{array}$ & $\begin{array}{l}\text { Stage } 3 \\
64 \mathrm{~nm} \\
\end{array}$ & $\begin{array}{l}\text { Stage } 4 \\
32 \mathrm{~nm} \\
\end{array}$ & $\begin{array}{l}\text { Stage } 5 \\
16 \mathrm{~nm} \\
\end{array}$ & $\begin{array}{l}\text { Main } \\
8 \mathrm{~nm} \\
\end{array}$ \\
\hline \multicolumn{7}{|l|}{ Radiator undulator } \\
\hline length of undulator, $\mathrm{m}$ & & 1.4 & 1.7 & 1.9 & 2 & \\
\hline period, $\mathrm{cm}$ & & 5 & 4.5 & 4 & 3.3 & \\
\hline peak field, $\mathrm{T}$ & & 1.1 & 0.94 & 0.75 & 0.65 & \\
\hline$K$-value & & 5.5 & 4 & 2.8 & 2 & \\
\hline \multicolumn{7}{|l|}{ Demodulator chicane } \\
\hline net momentum compaction, $\mathrm{mm}$ & & 0.3 & 0.3 & 0.3 & 0.03 & \\
\hline total chicane length, $\mathrm{m}$ & & 3 & 3 & 3 & 1.5 & \\
\hline lengh of each dipole magnet, $\mathrm{m}$ & & 0.25 & 0.25 & 0.25 & 0.25 & \\
\hline bend angle of each dipole, deg. & & 0.6 & 0.6 & 0.6 & 0.27 & \\
\hline magnetic field for each dipole, $\mathrm{T}$ & & 0.15 & 0.15 & 0.15 & 0.067 & \\
\hline \multicolumn{7}{|l|}{ FEL amplifier undulator } \\
\hline length of undulator, $\mathrm{m}$ & 1.95 & 1.6 & 2.4 & 2.8 & 4.6 & 13.1 \\
\hline period, $\mathrm{cm}$ & 6.5 & 5 & 4.5 & 4 & 3.3 & 2.73 \\
\hline peak field, $\mathrm{T}$ & 1.1 & 1.1 & 0.94 & 0.75 & 0.65 & 0.5 \\
\hline$K$-value & 6.8 & 5.5 & 4 & 2.8 & 2 & 1.26 \\
\hline \multicolumn{7}{|l|}{ Modulator chicane } \\
\hline net momentum compaction, $\mu \mathrm{m}$ & 18 & 7.5 & 3.5 & 1.5 & & \\
\hline total chicane length, $\mathrm{m}$ & 1.5 & 1.5 & 1.5 & 1.5 & & \\
\hline length of each dipole magnet, $\mathrm{m}$ & 0.25 & 0.25 & 0.25 & 0.25 & & \\
\hline bend angle of each dipole, deg. & 0.2 & 0.13 & 0.09 & 0.06 & & \\
\hline magnetic field for each dipole, $\mathrm{T}$ & 0.05 & 0.033 & 0.023 & 0.015 & & \\
\hline
\end{tabular}

\section{Estimation of space charge and coherent synchrotron radiation effects}

Let us begin with the analysis of the space charge effects in the modulated electron beam moving along the $z$ axis of planar undulator. Natural oscillations associated with the space charge force arising from a small displacement of charge can be derived in several different ways, but here straightforward elementary approach is used. Let the beam excitation be periodic, sinusoidal in $z$. Beam modulation wavenumber $k$ corresponds to the frequency $\omega=k \bar{v}_{z}$, where $\bar{v}_{z}=v-c K^{2} /\left(4 \gamma^{2}\right)$ is the longitudinal velocity of electrons with nominal energy $E_{0}$, averaged over undulator period. Let us introduce the coordinate $s=z-\bar{v}_{z} t$. The beam current $j_{z}$ is a periodic function of the phase $k s$ and can be expanded in a Fourier series:

$$
j_{z}=\sum_{n=1}^{\infty} j_{n} \cos \left(n k s+\psi_{n}\right)
$$

where $j_{n}=a_{n} j_{0}$ and $\psi_{n}$ are the amplitude and phase of the $n$th harmonic of the beam current density, $j_{0}$ is the beam current density, and $a_{n}$ is the amplitude of first harmonic of reduced beam current density. When the transverse size of the electron beam is large, $\sigma_{r}^{2} \gg c^{2} \gamma_{z}^{2} / \omega^{2}$, the longitudinal space charge field of the electron beam is defined by the local density modulation. In the framework of the one-dimensional model we can 


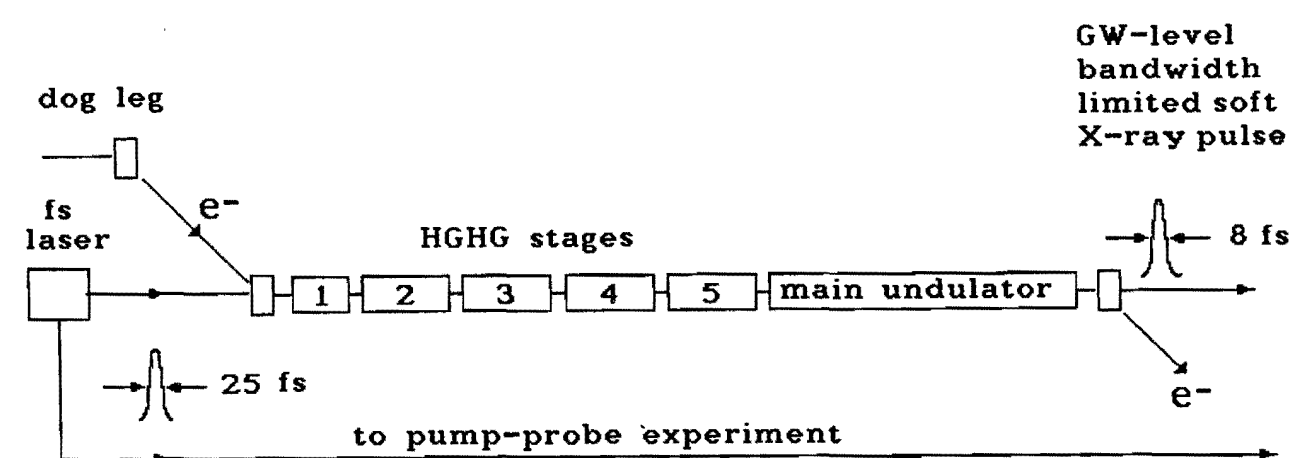

single light pulse produces fs soft $X$-ray pulse with

probability about $10 \%$

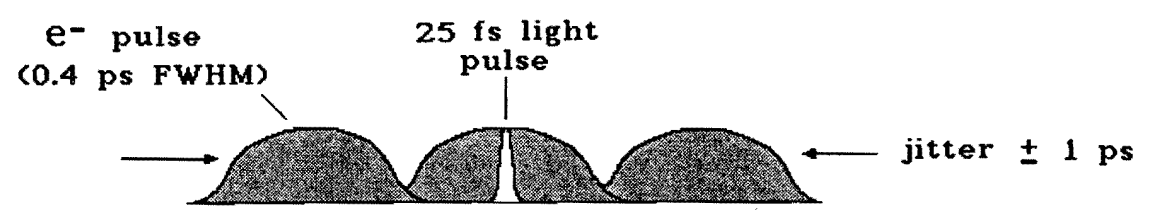

at the first stage entrance

Fig. 5. Up: Schematic layout of the femtosecond pump-probe facility at TTF. Down: Effect of a electron pulse jitter on the subharmonically seeded soft X-ray FEL if a single $25 \mathrm{fs}$ optical pulse is used as a seed signal

derive from Maxwell's equations the following expression for the longitudinal component of electric field:

$$
E_{z}=4 \pi \omega^{-1} \sum_{n=1}^{\infty} n^{-1} j_{n} \sin \left(n k s+\psi_{n}\right)
$$

The Lorentz force is used to derive the equations of motion of electrons with charge $(-e)$ and mass $m_{\mathrm{e}}$ in the space charge field. The rate of electron energy change is

$$
\frac{\mathrm{d} E}{\mathrm{~d} t}=m_{\mathrm{e}} c^{2} \frac{\mathrm{d} \gamma}{\mathrm{d} t}=-e \bar{v}_{z} E_{z}
$$

Remembering that $\mathrm{d} z=\bar{v}_{z} \mathrm{~d} t$, we find

$$
\frac{\mathrm{d} \gamma}{\mathrm{d} z}=-\frac{e}{m_{\mathrm{e}} c^{2}} E_{z}=-\frac{4 \pi j_{0} c a_{1}}{I_{\mathrm{A}} \omega} \sin \left(k s+\psi_{1}\right),
$$

where $I_{\mathrm{A}}=m_{\mathrm{e}} c^{3} / e \simeq 17 \mathrm{kA}$ is the Alfven current. In our case $a_{1} \ll 1$, and space charge fields are calculated using only the first harmonic of the electron beam density modulation. The amplitude $a_{1}$ and phase $\psi_{1}$ of the first harmonic of the beam current density are given by the expression

$$
a_{1} \exp \left(\mathrm{i} \psi_{1}\right)=-\frac{2}{N} \sum_{j=1}^{N} \exp \left(-\mathrm{i} k s_{j}\right)
$$


where $\left(k s_{j}\right)$ are the phases of the electrons within the phase interval $(0,2 \pi)$, and $N$ is the number of particles per wavelength.

For particles moving with nominal velocity $\bar{v}_{z}$ the phase $k s_{j}$ is constant along $z$. Therefore, $\mathrm{d}\left(k s_{j}\right) / \mathrm{d} z \neq 0$ only in the case when the particle energy deviates from the nominal value $\delta E=E-E_{0} \neq 0$. When the particle energy $E$ does not differ significantly from nominal value $E_{0}$, the derivative of phase $\left(k s_{j}\right)$ can be written as

$$
\frac{\mathrm{d}\left(k s_{j}\right)}{\mathrm{d} z}=\frac{k}{\bar{v}_{z}} \frac{\mathrm{d} \bar{v}_{z}}{\mathrm{~d} E}\left(E_{j}-E_{0}\right)=\frac{k}{\gamma_{z}^{2} E_{0}} \delta E_{j} .
$$

Now we differentiate derivative of phase with respect to $z$ and substitute $\mathrm{d}\left(\delta E_{j}\right) / \mathrm{d} z$. As a result, we obtain

$$
\frac{\mathrm{d}^{2}\left(k s_{j}\right)}{\mathrm{d} z^{2}}=-\frac{4 \pi j_{0} a_{1}}{\gamma_{z}^{2} \gamma I_{\mathrm{A}}} \sin \left(k s_{j}+\psi_{1}\right)
$$

Let us now introduce the notion of complex bunching

$$
\hat{a}_{1}=-\frac{2}{N} \sum_{j=1}^{N} \exp \left(-\mathrm{i} k s_{j}\right) .
$$

In the linear approximation one can find that the second derivative of complex bunching may be written in the form:

$$
\frac{\mathrm{d}^{2} \hat{a}_{1}}{\mathrm{~d} z^{2}}=\frac{\mathrm{d}^{2}}{\mathrm{~d} z^{2}}\left[\frac{2}{N} \sum_{j=1}^{N}\left(\mathrm{i} k s_{j}\right) \exp \left(-\mathrm{i} k s_{j}^{(0)}\right)\right]
$$

where $\left(k s_{j}^{(0)}\right)$ are the initial (unperturbed) phases. Substituting $\mathrm{d}^{2}\left(k s_{j}\right) / \mathrm{d} z^{2}$, we obtain the equation:

$$
\frac{\mathrm{d}^{2} \hat{a}_{1}}{\mathrm{~d} z^{2}}+k_{\mathrm{p}}^{2} \hat{a}_{1}=0
$$

where

$$
k_{\mathrm{p}}=\left[\left(4 \pi j_{0}\right) /\left(\gamma_{z}^{2} \gamma I_{\mathrm{A}}\right)\right]^{1 / 2}
$$

is the wavenumber of the longitudinal plasma oscillations.

Now, we examine what happens if the beam is initially excited at the density perturbation amplitude $a_{1}$. During plasma oscillation, the energy modulation grows from zero to some maximal amplitude $(\delta \gamma)_{\max }$ at a distance $z=\pi /\left(2 k_{\mathrm{p}}\right)$. After integration of $\mathrm{d} \gamma / \mathrm{d} z$ we obtain a peak energy deviation of

$$
(\delta \gamma)_{\max }=\frac{4 \pi j_{0} c a_{1}}{I_{\mathrm{A}} k_{\mathrm{p}} \omega}=\left[\frac{4 \pi j_{0} \gamma}{I_{\mathrm{A}}}\right]^{1 / 2} \frac{c \gamma_{z} a_{1}}{\omega} .
$$


The next problem to be studied is that of estimating the space charge effects influencing the operation of the subharmonically seeded FEL at TTF. In the previous section we have considered the HGHG scheme when each stage (except the first) is equipped with demodulator. Such design is safe with respect to the problem of plasma oscillations. Now we can estimate the operation of HGHG FEL without demodulators. In this case, following the first stage, the beam immediately enters FEL amplifier of the second stage. Upon passing through the FEL amplifier, electron beam modulation at $130 \mathrm{~nm}$ is amplified exponentially. The value of the first $(260 \mathrm{~nm})$ harmonic density modulation exiting the first stage is about $10 \%$. Upon passing through the second FEL amplifier the electric field of the space charge induces the additional energy modulation at $260 \mathrm{~nm}$ wavelength. Let us do some estimations now. The parameters of the electron beam in our case are: relativistic factor $\gamma \simeq 2000$, undulator parameter of second $(130 \mathrm{~nm})$ FEL amplifier $K=5.4$, longitudinal relativistic factor for the second FEL amplifier undulator $\gamma_{z} \simeq$ 500 , current density $j_{0} \simeq I /\left(2 \pi \sigma_{r}^{2}\right)$, peak current $I \simeq 2.5 \mathrm{kA}$, rms transverse beam size $\sigma_{r} \simeq 70 \mu \mathrm{m}$, wavelength of density modulation $\lambda=2 \pi c / \omega \simeq 260 \mathrm{~nm}$, amplitude of density modulation $a_{1} \simeq 0.1$. Calculation of the plasma wavenumber and peak energy deviation gives us the following results:

$$
\begin{gathered}
k_{\mathrm{p}} \simeq(2.5 \mathrm{~m})^{-1}, \\
m_{\mathrm{e}} c^{2}(\delta \gamma)_{\max } \simeq 0.4 \mathrm{MeV} .
\end{gathered}
$$

At the end of the first stage there is the energy modulation $\delta E \simeq 0.25 \mathrm{MeV}$. Comparing this value with the space charge induced energy modulation in the second undulator $(0.4$ $\mathrm{MeV}$ ), we can conclude that to make preservation of the energy spread in the case of the subharmonically seeded FEL operation without demodulators is more difficult. It should be noted that following the second stage the beam, modulated at $260 \mathrm{~nm}$, enters the third FEL amplifier undulator where longitudinal relativistic factor $\gamma_{z}$ is larger and, according to expression for $(\delta \gamma)_{\max }$, longitudinal space charge electric field would induce larger energy modulation at $260 \mathrm{~nm}$ than in the second stage. As a result, the small induced energy spread requirement dictate the use of demodulator chicane in 2nd-5th stages.

Let us now estimate the coherent synchrotron radiation (CSR) effect. Particles in a modulated bunch following a curved path may radiate coherently at a wavelength of the beam density modulation. The radiated power is proportional to the square of the current, hence proportional in this case to $N^{2} a_{1}^{2}$, where $N$ is the number of particles per wavelength of density modulation. When an electron bunch passes the demodulator dispersion sections, radiative interaction induces an additional energy spread in the electron beam. The problem connected with radiative interaction of the particles in the line-charge bunch with sinusoidal density excitation moving in a bending magnet has been investigated analytically $[16,17]$. If we neglect the beam transverse size effects, the CSR induced energy spread is predicted to be roughly

$$
(\delta \gamma)_{\max } \simeq \frac{2 \Gamma(2 / 3) I a_{1} L_{\mathrm{m}}}{3^{1 / 3} I_{\mathrm{A}}}\left[\frac{\omega}{c R^{2}}\right]^{1 / 3}=1.88 \frac{I a_{1} L_{\mathrm{m}}}{I_{\mathrm{A}}}\left[\frac{\omega}{c R^{2}}\right]^{1 / 3}
$$


where $L_{\mathrm{m}}$ and $R$ are the chicane bending magnet length and bending radius, $\Gamma(\cdots)$ is the gamma function. The point where beam transverse size becomes important can be estimated as $c / \omega>\sigma_{r}^{3 / 2} / R^{1 / 2}$. Let us present some estimations. The design of demodulator chicane is based on the need to minimize CSR induced energy spread. The parameters of the demodulator chicane in our case are: $L_{\mathrm{m}}=25 \mathrm{~cm}$, bending radius $R=25 \mathrm{~m}$ $\left(\theta=0.6^{\circ}\right)$. Calculation of the peak energy deviation for the first demodulator (in this case $2 \pi c / \omega=260 \mathrm{~nm}$ ) gives the following result:

$$
m_{\mathrm{e}} c^{2}(\delta \gamma)_{\max } \simeq 0.15 \mathrm{MeV}
$$

This means that CSR effects should not be a serious limitation for the adopted design.

\section{Estimation of essential shot noise effects on basis of a simple model}

As for the HGHG FEL operating in the short wavelength range, its noise properties are defined only by the shot noise. To describe the noise output quantitatively, we should define the quality criterion of the HGHG FEL. One possible definition can be made as follows. A HGHG FEL can be characterized by a noise factor $F$ that related the input to output signal to noise ratio:

$$
F=\frac{\left(P_{\mathrm{s}} / P_{\mathrm{n}}\right)_{\text {in }}}{\left(P_{\mathrm{s}} / P_{\mathrm{n}}\right)_{\text {out }}}
$$

where $P_{\mathrm{s}}$ and $P_{\mathrm{n}}$ are the power of signal and noise, respectively. It is relevant to comment on the definitions of output and input power of signal and noise. Because the signal bandwidth is much less than the noise bandwidth, we can easily select HGHG FEL output signal and noise radiation originating from different frequency regions of radiation spectrum. It is natural to describe the input signal and noise power by the radiation power of signal and noise at the first FEL amplifier exit. The actual physical picture of shot noise takes into account that the fluctuations of the current density in the electron beam are uncorrelated in time and space. Thus, a large number of transverse radiation modes are excited when the electron beam enters the undulator. As a rule, the first FEL amplifier has gain of about 10-20 dB only which is insufficient for transverse mode selection. Nevertheless, for a long last (main) undulator the only fundamental TEM To $_{00}$ mode, which has maximal gain, should survive. That is why the input noise power $\left(P_{\mathrm{n}}\right)_{\mathrm{in}}$ should be treated as a contribution to fundamental radiation mode only.

An intrinsic disadvantage in the short wavelength HGHG FEL is the enormous value of noise factor. This is the direct effect of the frequency multiplication. The dependence of a noise factor can be given as a function of a frequency multiplication factor $N$. Successive multiplication to the $N$ th harmonic resulting in at least $N^{2}$ - fold increasing of the noise factor

$$
F>N^{2} \text { at } \omega_{0} \rightarrow n_{1} \omega_{0} \rightarrow n_{1} n_{2} \omega_{0} \rightarrow \cdots \rightarrow N \omega_{0}
$$


as one can expect from simple physical consideration (see below). This fundamental result is of great practical importance, because a crucial condition in HGHG FEL is that the output signal to noise ratio $\left(P_{\mathrm{s}} / P_{\mathrm{n}}\right)_{\text {out }}$ must be made much larger than unity, in order for the properties of the output radiation to be a map of the characteristics of the high-quality seed laser.

Simple physical consideration can lead directly to a crude approximation for the value of $F$. It should be noted that the method which can be applied to determine the output field perturbation is independent of a specific kind of HGHG technique. This calculation depends on the frequency multiplication factor $N$ only. The field of amplified electromagnetic wave in the first FEL amplifier can be represented as

$$
E_{1}=E_{\mathrm{s}} \exp \left(\mathrm{i} \omega_{0} t\right)+\sum_{j} u_{j} \exp \left(\mathrm{i} \omega_{0} t+\Delta \omega_{j} t\right)+\text { C.C. },
$$

where $E_{\mathrm{s}}$ is the amplitude of amplified seed signal. The quantities $u_{j} \exp \left(\mathrm{i} \omega_{0} t+\mathrm{i} \Delta \omega_{j} t\right)$ represent the (small) signal changes due to the shot noise. Starting with a field at fundamental harmonic in the first stage (2) and omitting an inessential common factor, we find for the field in the main undulator at frequency $N \omega_{0}$ :

$$
E_{\mathrm{N}}=E_{\mathrm{s}}^{N} \mathrm{e}^{\mathrm{i} N \omega_{0} t}+N E_{\mathrm{s}}^{N-1} \mathrm{e}^{\mathrm{i}(N-1) \omega_{0} t} \sum_{j} u_{j} \mathrm{e}^{\mathrm{i} \omega_{0} t} \mathrm{e}^{\Delta \omega_{j} t}+\text { C.C. },
$$

where we have assumed that at the end of each stage the amplitude of a higher harmonic density modulation is small. It is obvious, this is the situation that is encountered in any HGHG scheme. As we have seen in Sec. 2, the amplitude of the $n$th harmonic of the beam density modulation, $a_{n}$, is proportional to the $n$th power of the field amplitude at the fundamental frequency, i.e. $a_{n} \propto\left(E_{1}\right)^{n}$ at $a_{n} \ll 1$. Therefore, the field amplitude $E_{N}$ in the main undulator is proportional to $E_{N} \propto a_{N} \propto\left(E_{1}\right)^{N}$. When derived (3), we also required the output signal to noise ratio to be much larger than unity. These two assumptions are quite general and do not reduce significantly the practical applicability of the result obtained. However, for simplicity presented derivation is limited to the case where only first FEL amplifier have significant contribution to the noise output.

In the frame of approximations discussed above the output signal to noise ratio can be represented as

$$
\left(\frac{P_{\mathrm{s}}}{P_{\mathrm{n}}}\right)_{\text {out }}=\frac{\left\langle\left(E_{1}^{N} \mathrm{e}^{\mathrm{i} N \omega_{0} t}+\text { C.C. }\right)^{2}\right\rangle}{\left\langle\left(N E_{1}^{N-1} \mathrm{e}^{\mathrm{i}(N-1) \omega_{0} t} \sum_{j} u_{j} \mathrm{e}^{\mathrm{i} \omega_{0} t} \mathrm{e}^{\Delta \omega_{j} t}+\text { C.C. }\right)^{2}\right\rangle},
$$

where symbol $\langle\cdots\rangle$ means the ensemble average over bunches. Thus, frequency multiplication by $N$ degrades the signal/noise ratio by $N^{2}$ :

$$
\left(\frac{P_{\mathrm{s}}}{P_{\mathrm{n}}}\right)_{\text {out }}=\frac{1}{F}\left(\frac{P_{\mathrm{s}}}{P_{\mathrm{n}}}\right)_{\text {in }}=\frac{1}{N^{2}}\left(\frac{P_{\mathrm{s}}}{P_{\mathrm{n}}}\right)_{\text {in }} .
$$

Here we illustrated the essential shot noise effects on the basis of a simple model. Indeed, the present derivation assumes shot noise influence in the 2nd, 3rd and other stages to 
be negligible compared to the shot noise influence in the 1st stage. Nevertheless, the problem of calculation of HGHG FEL noise factor is more complicated and there are situations where, for instance, shot noise in the second stage can provide comparable or even larger output signal perturbation than shot noise in the first stage. As a result, we can conclude only that in practical situations noise factor satisfies inequality $F>N^{2}$. Complete description of the noise output of HGHG FEL can be performed only with numerical simulation codes. The specific numerical example of subharmonically seeded soft X-ray FEL, which we present below, illustrates the complexity of shot noise effects in a HGHG FEL.

\section{Numerical study of the harmonic generation process in a multistage HGHG FEL}

Numerical example for multistage HGHG FEL is given for project parameters of the electron beam at the TESLA Test Facility (see Table 1). FEL project at the TTF will proceed in two stages. The first stage assumes running conventional SASE FEL scheme, and at the second stage it will be upgraded into the self-seeding option. General parameters for these two options are given in Tables 4 and 5. Figure 6 presents typical temporal and spectral structure of the radiation pulse.

Table 4

Parameters of conventional SASE FEL at TTF

/ Undulator with separate focusing system /

\begin{tabular}{|lcccc|}
\hline & Units & $6.4 \mathrm{~nm}$ & $13 \mathrm{~nm}$ & $30 \mathrm{~nm}$ \\
\hline Undulator $^{*}$ & & & & \\
Period & $\mathrm{cm}$ & & 2.73 & \\
Gap & $\mathrm{mm}$ & & 12 & \\
Peak magnetic field & $\mathrm{T}$ & & 0.495 & \\
External $\beta$-function & $\mathrm{m}$ & & 4.5 & \\
rms beam size & $\mu \mathrm{m}$ & 68 & 80 & 100 \\
Output radiation & & & & \\
\hline Wavelength & $\mathrm{nm}$ & 6.4 & 13 & 30 \\
Saturation length & $\mathrm{m}$ & 26.2 & 21.2 & 17.8 \\
Peak power & $\mathrm{GW}$ & 2.8 & 2.5 & 1.8 \\
Average power & $\mathrm{W}$ & 40 & 35 & 27 \\
Spot size at the undulator exit (FWHM) & $\mu \mathrm{m}$ & 190 & 220 & 270 \\
Angular divergence (FWHM) & $\mu \mathrm{rad}$ & 24 & 40 & 70 \\
Bandwidth (FWHM) & $\%$ & 0.36 & 0.46 & 0.62 \\
Pulse duration (FWHM) & $\mathrm{fs}$ & 200 & 200 & 200 \\
Peak flux of photons & $\# / \mathrm{sec}$ & $9 \times 10^{25}$ & $1.6 \times 10^{26}$ & $2.8 \times 10^{26}$ \\
Average flux of photons & $\# / \mathrm{sec}$ & $1.3 \times 10^{18}$ & $2.3 \times 10^{18}$ & $4 \times 10^{18}$ \\
Peak spectral brilliance & $B^{* *}$ & $2.4 \times 10^{30}$ & $8.1 \times 10^{29}$ & $2 \times 10^{29}$ \\
Average spectral brilliance & $B^{* *}$ & $3.5 \times 10^{22}$ & $1.2 \times 10^{22}$ & $2.9 \times 10^{21}$ \\
\hline
\end{tabular}

${ }^{*}$ Hybrid, planar undulator.

** In units of photons $/ \mathrm{sec} / \mathrm{mrad}^{2} / \mathrm{mm}^{2} /(0.1 \%$ bandwidth $)$. 
Table 5

Parameters of self-seeding SASE FEL at TTF

\begin{tabular}{|ll|}
\hline Undulator & Planar \\
Type & $2.73 \mathrm{~cm}$ \\
Period, $\lambda_{\mathrm{w}}$ & $4.97 \mathrm{kGs}$ \\
Peak magnetic field, $H_{\mathrm{w}}$ & $13 \mathrm{~m}$ \\
Length of 1st undulator & $17 \mathrm{~m}$ \\
Length of 2nd undulator & $300 \mathrm{~cm}$ \\
External $\beta$-function, & $57 \mu \mathrm{m}$ \\
rms transverse beam size & \\
Output radiation & $6.4 \mathrm{~nm}$ \\
Wavelength, $\lambda$ & $7 \times 10^{-5}$ \\
Bandwidth, $\Delta \lambda / \lambda$ & $15 \mu \mathrm{rad}$ \\
rms angular divergence & $90 \mu \mathrm{m}$ \\
rms spot size & $100 \mathrm{fs}$ \\
Time of coherence, $\tau_{\mathrm{c}}$ & $2 \mathrm{GW}$ \\
Power average over pulse & $0.6 \mathrm{~mJ}$ \\
Flash energy & $5 \%$ \\
Fluctuations of the flash energy & $40 \mathrm{~W}$ \\
Average power & \\
\hline
\end{tabular}

Despite conventional SASE FEL is capable to provide much higher peak brilliance than synchrotron radiation sources, it still possesses a high potential for its further increase. Mainly brilliance of the output radiation from the SASE FEL is limited by poor longitudinal coherence of the radiation: it consists of a large number of statistically independent wavepackets (see Fig. 6). This is natural consequence of the start-up from shot noise of the FEL amplification process. An improvement of the longitudinal coherence is possible only when coherent radiation from external source is fed to the undulator entrance. The power of the seed radiation must exceed significantly effective power of shot noise in the electron beam in order to obtain fully coherent radiation at the exit of the FEL amplifier. The value of effective shot noise power is relatively high. Typically it is in the range from about one hundred Watt (FEL of optical wavelength range) up to few kilowatts (an X-ray FEL). Presently available coherent radiation sources allow to solve the seeding problem only for FELs operating in optical wavelength range.

Two-stage (or, self-seeding) SASE FEL scheme provides the possibility of effective seeding [3]. This scheme consists of two undulators and a monochromator located between them. Modern techniques allows one to design and manufacture monocromators with required parameters which are capable to cover wavelength range from an optical down to an X-ray band. The spectral bandwidth of the radiation from two-stage SASE FEL is defined by the finite duration of the electron pulse. The shot-to-shot fluctuations of energy spectral density are dramatically reduced in comparison with the $100 \%$ fluctuations in a SASE FEL [4]. The peak and average brilliance are by two orders of magnitude higher than the values which could be reached by a conventional X-ray SASE FEL (see Tables 4 and 5) The price for a better quality of output radiation is increase of the scale of the facility. First, the total undulator length should be of about 1.5 times larger than for conventional scheme. Second, monochromator and bypass for the electron beam require 

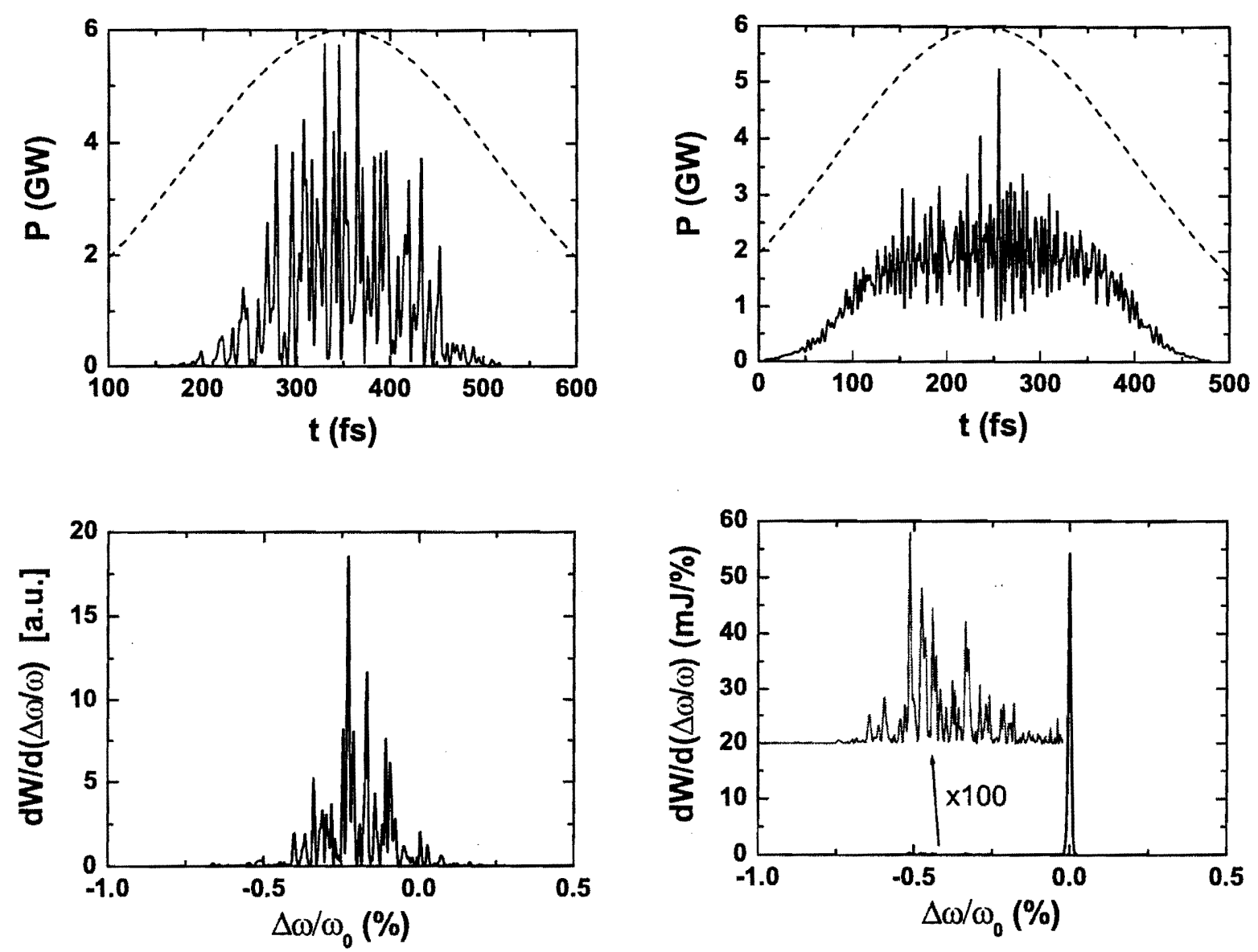

Fig. 6. Temporal and spectral structure of the radiation pulse from conventional SASE FEL (left column) and self-seeding FEL (right column). Dashed curves denote longitudinal profile of the electron bunch.

an additional space (in the case of TTF FEL it is about 22 meters).

In this section we analyze possibilities of the HGHG FEL scheme described in the previous sections. To be specific, we consider numerical example for its possible realization at the TESLA Test Facility at DESY. However, this specific example highlights all general properties of the HGHG FEL schemes.

We performed simulations for two options of HGHG schemes which differ by the pulse duration of the seed laser only. The first option corresponds to a long seed laser pulse, 5 ps FWHM, and the second option illustrates properties of the HGHG scheme when it is seeded by the laser pulse of $30 \mathrm{fs}$ duration (FWHM). General parameters of the laser system are presented in Table 2. Parameters of magnetic system are summarized in Table 3.

Numerical results presented in this paper are obtained with a version of code FAST upgraded for simulation of higher harmonics. This code allows one to perform threedimensional simulations of the FEL process taking into account diffraction, space-charge, energy spread and slippage effects, and shot noise in the electron beam. An electron bunch of any transverse and longitudinal profile can be simulated. General features of the code 

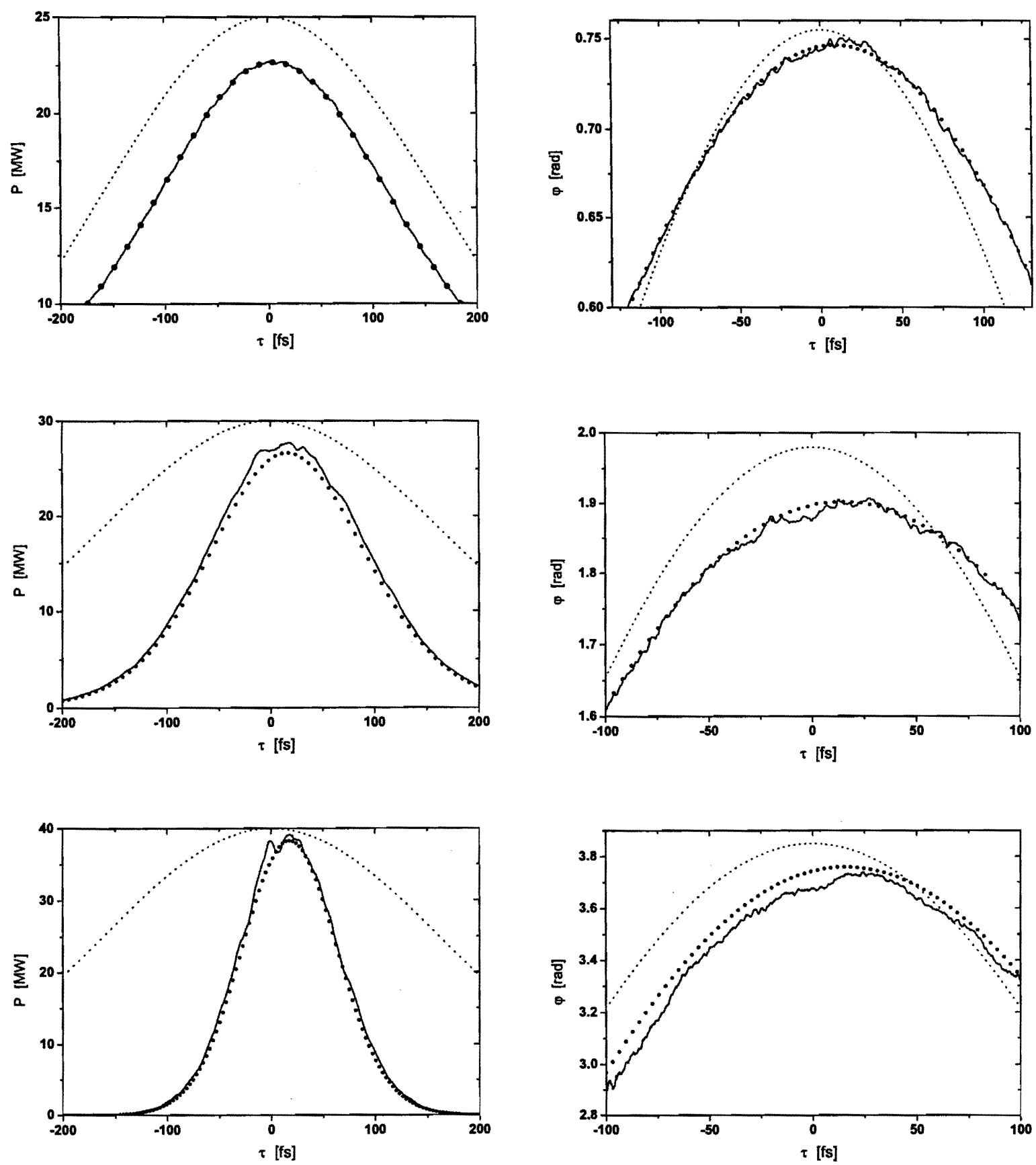

Fig. 7. Time structure of radiation power (left column) and phase of output radiation (right column) at the exit of different stages of HGHG scheme (upper, middle, and lower plots correspond to the 1st, 2nd, and 3rd stage, respectively). Solid curves are calculated taking into account shot noise in the electron beam, and the circles present the results without noise effect. Dashed curves denote longitudinal profile of the electron bunch. The first stage is seeded by a long laser pulse.

FAST are described in [19]. In the present simulations we assumed both transverse and longitudinal profile of the electron beam to be gaussian.

The simulation procedure was organized as follows. Calculations of the full HGHG 

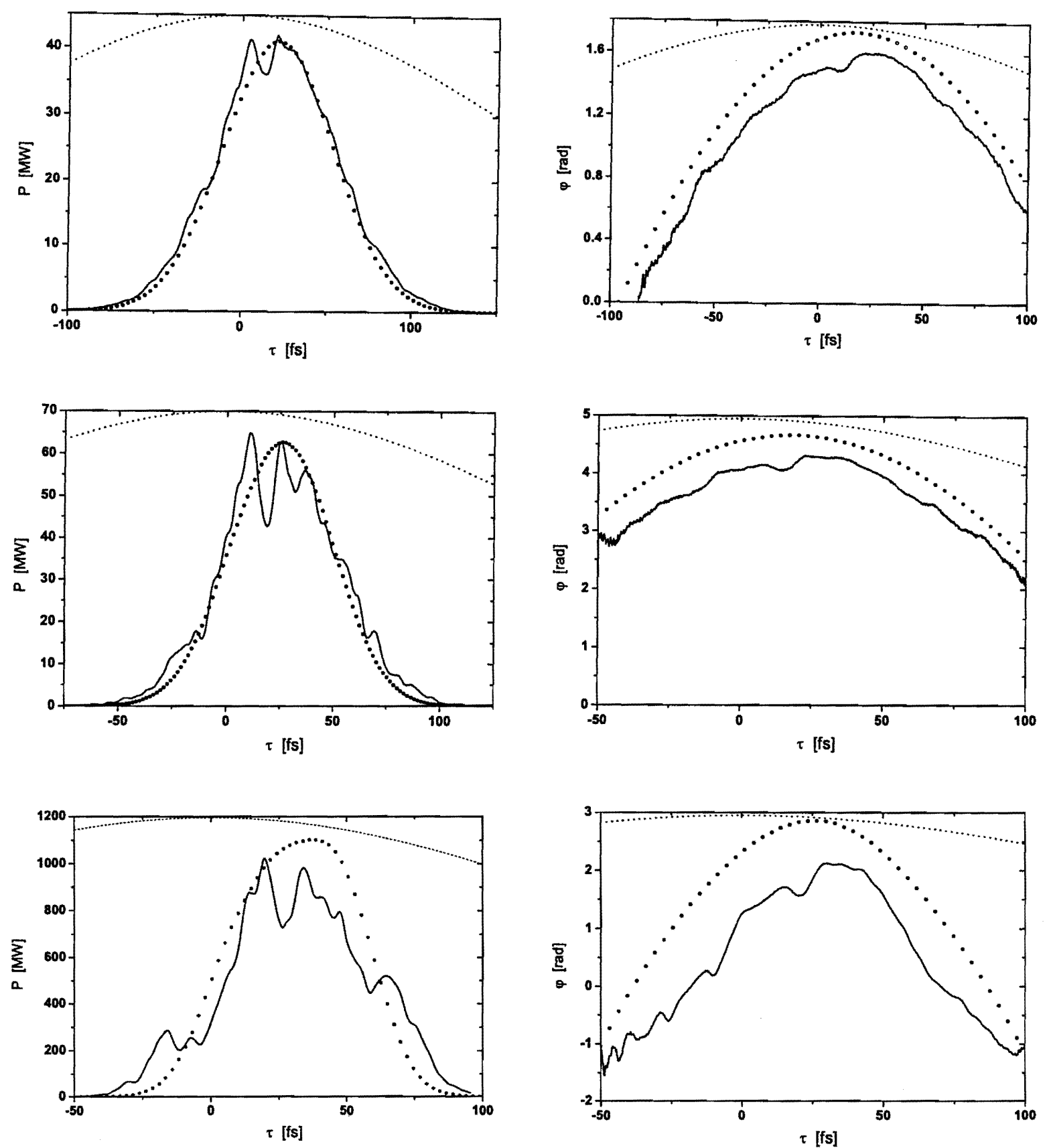

Fig. 8. Time structure of radiation power (left column) and phase of output radiation (right column) at the exit of different stages of HGHG scheme (upper, middle, and lower plots correspond to the 4 th and 5 th stages, and main undulator, respectively). Solid curves are calculated taking into account shot noise in the electron beam, and the circles present the results without noise effect. Dashed curves denote longitudinal profile of the electron bunch. The first stage is seeded by a long laser pulse.

scheme have been performed within one simulation run. I.e. we kept the same simulation mesh for the whole length of the system. This allowed us to avoid any artificial simulation effects which usually appear during recalculation of input parameters between different simulation runs. In particular, this precaution is extremely important for time-dependent 

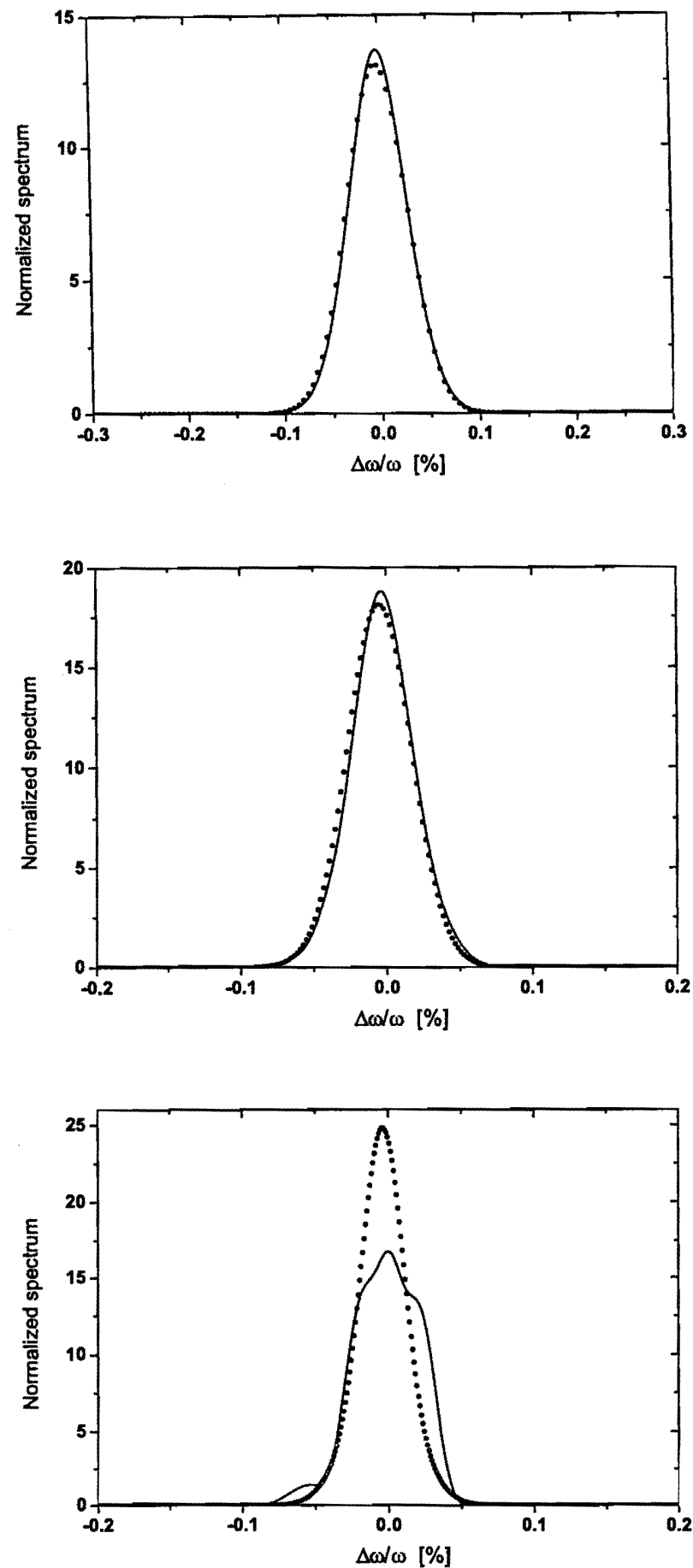

Fig. 9. Spectrum of output radiation at the exit of different stages of HGHG scheme (upper, middle, and lower plots correspond to the 4th and 5th stages, and main undulator, respectively). Solid curves are calculated taking into account shot noise in the electron beam, and the circles present the results without noise effect. The first stage is seeded by a long laser pulse.

simulations taking into account shot noise in the electron beam. The steps of the simulation procedure are as follows: 


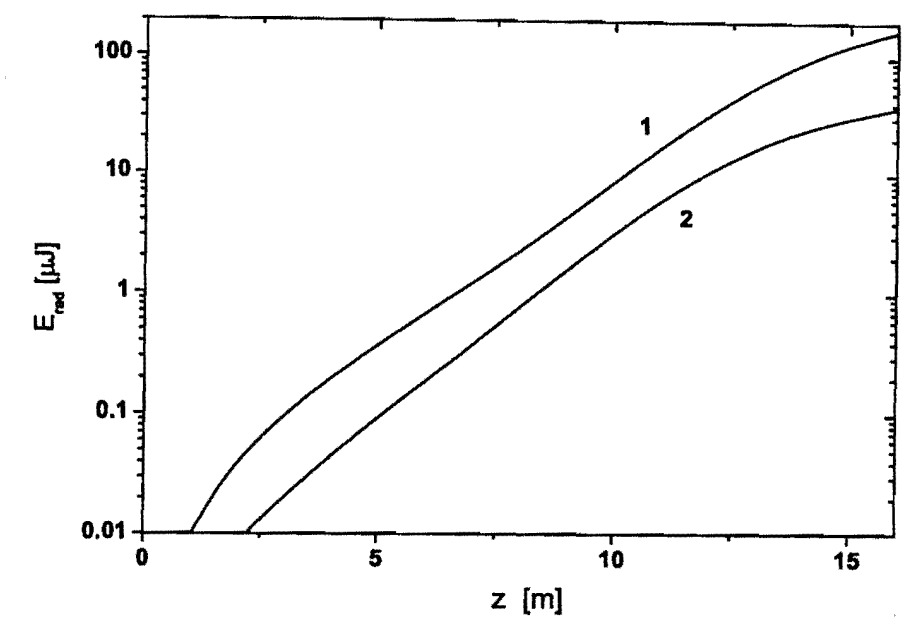

Fig. 10. Energy in the radiation pulse versus the length of the undulator of the 6 th stage. Curves 1 and 2 correspond to a long ( 5 ps FWHM) and a short ( $30 \mathrm{fs}$ FWHM) laser seeding pulse.

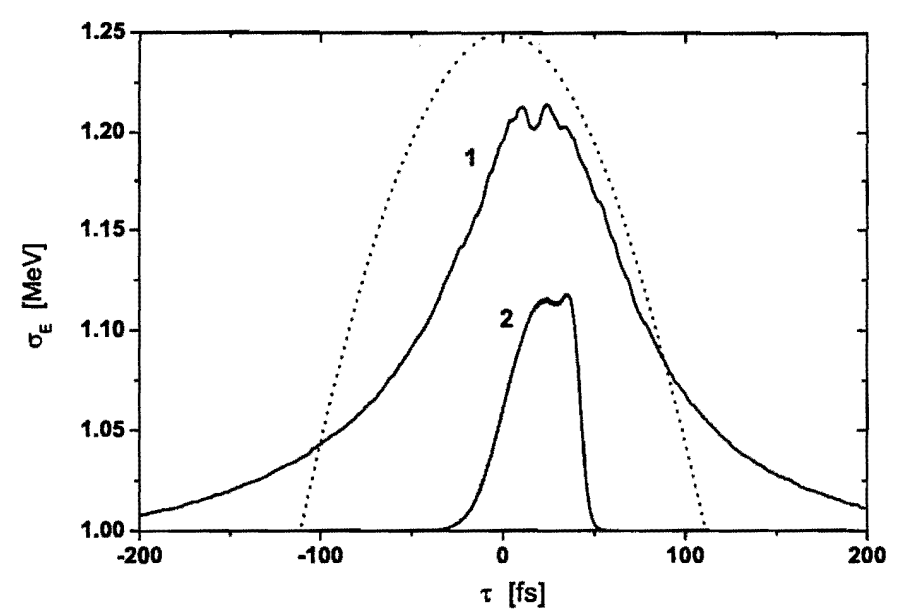

Fig. 11. Energy spread in the electron bunch at the exit of the 5 th stage. Curves 1 and 2 correspond to a long ( 5 ps FWHM) and a short (30 fs FWHM) laser seeding pulse. Dashed curve denotes longitudinal profile of the electron bunch.

(1) The HGHG scheme starts from conventional FEL amplifier amplifying the signal from external laser ( $256 \mathrm{~nm}$ wavelength). Maximum output power at the exit of the FEL amplifier is about $25 \mathrm{MW}$. Output radiation contains a contribution of the shot noise which is transported with the radiation and is amplified in the proceeding stages of the HGHG scheme.

(2) The macroparticle ensemble is traced through the modulator (dispersion section). At the exit of this stage the code produces macroparticle ensemble which distribution contains the second harmonic of the beam density modulation (natural shot noise in the electron beam and that component which has been induced by the bunching process in the modulator). The value of the induced modulation of the second harmonic (on the top of shot noise) is equal to 0.03 in the center of the electron beam. 

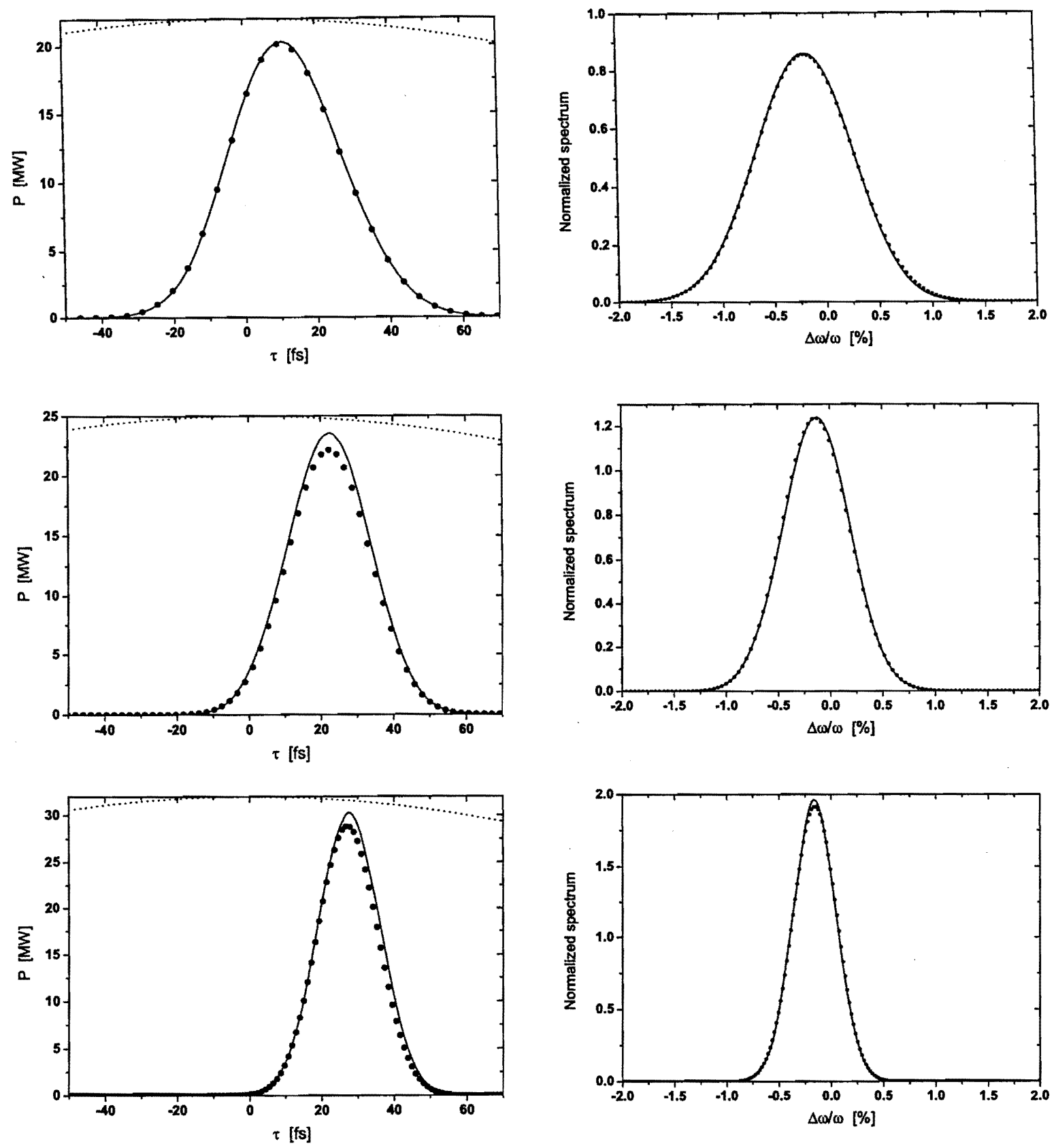

Fig. 12. Time structure of radiation power (left column) and spectrum of output radiation (right column) at the exit of different stages of HGHG scheme (upper, middle, and lower plots correspond to the 1st, 2nd, and 3rd stage, respectively). Solid curves are calculated taking into account shot noise in the electron beam, and the circles present the results without noise effect. Dashed curves denote longitudinal profile of the electron bunch. The first stage is seeded by a short ( $30 \mathrm{fs}$ FWHM) laser pulse.

(3) The macroparticle ensemble enters the radiator undulator resonant at the wavelength of the second harmonic, $128 \mathrm{~nm}$. Maximum value of the output power at the exit of the radiator undulator is about $1 \mathrm{MW}$. The noise contribution to the output power is increased further due to adding shot noise effects in the radiator undulator.

(4) The next stage is demodulator which suppresses modulation induced in the electron 

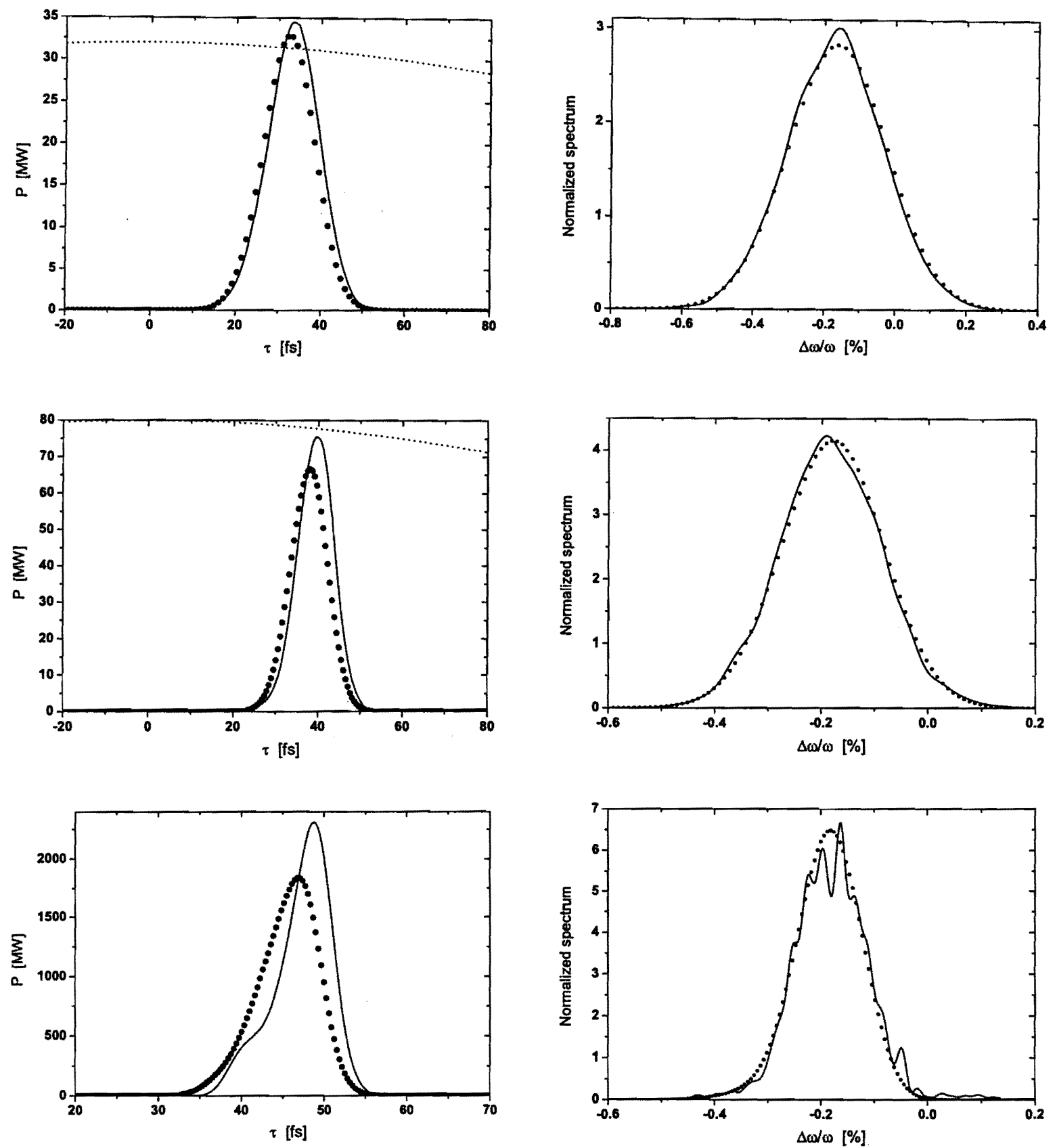

Fig. 13. Time structure of radiation power (left column) and spectrum of output radiation (right column) at the exit of different stages of HGHG scheme (upper, middle, and lower plots correspond to the 4th and 5 th stages, and main undulator, respectively). Solid curves are calculated taking into account shot noise in the electron beam, and the circles present the results without noise effect. Dashed curves denote longitudinal profile of the electron bunch. The first stage is seeded by a short ( $30 \mathrm{fs}$ FWHM) laser pulse.

beam in the previous stages. The correlated energy modulation transforms to increase of uncorrellated energy spread. At this stage fresh ensemble of macroparticles is generated taking into account increase of the energy spread due to FEL process in the previous undulator and shot noise in the electron beam. 
(5) The beam enters the FEL amplifier undulator resonant at the wavelength of $128 \mathrm{~nm}$. The seeding signal for this amplifier consists of two contributions: radiation produced in the radiator undulator (which contains noise contribution developed in the previous undulators), and the shot noise in the electron beam at the wavelength of $128 \mathrm{~nm}$. Maximal value of the radiation power at the exit of this stage is about $25 \mathrm{MW}$.

(6) The simulations procedure continues as it is described starting from item (2) until the wavelength reaches the value of $8 \mathrm{~nm}$.

(7) Finally, the FEL process is calculated until saturation level in the main undulator.

So, the described algorithm gives a clear idea on the simulation procedure. Let us turn now to analysis of obtained results. All numerical results are illustrated for two cases: with and without taking into account shot noise in the electron beam. We start with the case of a long seeding optical pulse, 5 ps FWHM (see Figs. 7-11). When we trace the behaviour of an idealized case (without taking into account shot noise), we obtain perfect quality radiation pulse (both in temporal and spectral domain) at the exit of HGHG FEL. However, taking into account noise effect results in a degradation of the quality of the radiation pulse from stage to stage. As we mentioned in the previous section, the HGHG FEL scheme, as well as any frequency multiplication scheme possesses significant intrinsic disadvantage. Namely, contribution of the shot noise power to the signal grows quadratically with the number of harmonic to be generated. The results of numerical simulations are in good agreement with simple physical estimations presented in the previous section. The growth of the noise contribution is clearly illustrated with the plots for the phase of the radiation field (see Figs. 7 and 8). The plots for radiation spectrum are presented in Fig. 9. Analysis of the present numerical example shows that the shot noise in the first stage gives relatively small contribution to the final value of the noise to signal ratio at the exit of the HGHG scheme. This is achieved by means of increase of the power of seed radiation. However, HGHG scheme does not allow to do this for the next stages when a small beam density modulation serves as input signal. In our case the main contribution of noise degradation comes from the second stage. The third stage also gives visible contribution to the noise.

The most critical issue of the single-bunch HGHG scheme is growth of uncorrelated energy spread. However, parameters of the stages can be optimized in such a way that this effect almost does not lead to degradation of output radiation. Figure 11 illustrates the energy spread in the electron bunch at the entrance to the main undulator. It is seen that the increase of uncorrelated energy spread is $20 \%$ only.

An attractive feature of the HGHG scheme is that it is capable to produce shorter pulses than the self-seeding option (see Table 5 and Fig. 6 ). Even with a long seeding pulse the FWHM pulse duration of output radiation is about $80 \mathrm{fs}$. It is at least twice shorter than that produced by conventional SASE FEL (see Fig. 6). We performed a dedicated study of capabilities of HGHG scheme seeded by a short seeding pulse ( $30 \mathrm{fs}$ FWHM). Our calculations show that output signal has pulse duration of about $10 \mathrm{fs}$ FWHM. Of course, the noise degradation also takes place as it is seen from Figs. 12 and 13. One can wonder why the peak output power of the HGHG FEL seeded by a short pulse is larger with respect to the HGHG FEL seeded by a long pulse. This is not a general feature of the HGHG FEL scheme, but a natural consequence of the specific single-bunch scheme proposed in this paper. Explanation of discussed phenomenon comes from analysis of the 
plots for the energy spread in the electron beam at the exit of the HGHG FEL scheme (see Fig. 11). Degradation of the energy spread is less for a short seeding pulse because of slippage effect. At each stage the working area of the electron bunch slips forward onto less perturbed part of the electron bunch. Our calculations shows that peak power of HGHG FEL seeded by a long pulse can be improved by shifting in the main undulator the working area a little bit forward along the electron bunch.

The results presented above allows us to make conclusion that shot noise degradation does not allow to reach very short wavelengths with the HGHG FEL scheme. A limit imposed by this fundamental effect seems to be around $8 \mathrm{~nm}$ when HGHG procedure starts from UV seeding laser (multiplication factor of about 30). Practical limit is reached for even longer wavelength. The reason for this is that HGHG FEL scheme is extremely sensitive to fluctuations of the beam and seeding radiation parameters.

\section{Discussion}

In conclusion we would like to discuss some general aspects of noise influence on the HGHG FEL operation. It should be emphasized that despite the theory of HGHG FEL was developed over a decade, there are no papers devoted to the analysis of the noise properties of these sources. Here, it is relevant to remember that the analysis of the frequency multiplier chains and their effects on source noise in radar and similar systems has always been an important problem. The majority of communication and radar engineers are familiar with the fact that inserting the amplifier prior to frequency multiplication has the disadvantage that the phase noise contribution of the amplifier is multiplied by $n^{2}$, where $n$ is a frequency multiplication factor (see, for example, [20]). In the case of HGHG FEL this means that the effect of frequency multiplication by a factor of $N$ multiplies the first FEL amplifier noise power to carrier ratio by $N^{2}$. This prevents operation of HGHG FEL at very short wavelength range. The results presented in this paper have demonstrated that the HGHG FEL approach is quite adequate for a 10-100 $\mathrm{nm}$ coherent light source, but not scalable to an X-ray device.

Let us give a more detailed discussion of problems relating to the X-ray HGHG FEL noise output. Frequency multiplication process produces a noise degradation proportional to at least the square of the multiplication ratio. As a result, HGHG FEL starting from optical wavelength range $\left(\lambda_{\text {in }}>2000 \AA\right)$ cannot produce coherent radiation spanning to Angstrom wavelength range. The main problem is that the contribution of the noise to the output power increases drastically when approaching the X-ray band. Consider firstly an idealized case where only the first FEL amplifier has significant contribution to the noise output. Then the signal to noise ratio after an ideal frequency multiplier chain is smaller than the signal to noise ratio at the fundamental frequency by a factor of $F \simeq N^{2} \simeq 10^{7}$ at $\lambda_{\text {out }} \simeq 1 \AA$. If we want to make the ratio $\left(P_{\mathrm{s}} / P_{\mathrm{n}}\right)_{\text {out }}$ much larger than unity, $\left(P_{\mathrm{s}} / P_{\mathrm{n}}\right)_{\text {in }}$ must reach values of about $10^{9}$. To estimate the required value of the peak power of the seed laser pulse it is convenient to introduce the notion of an effective power of shot noise $P_{\text {sl }}$, which is usually used for numerical simulation of the SASE FEL with steady-state codes (see for example [18]). For the visible range of the spectrum the effective shot noise power for usual SASE FEL parameters is about $P_{\text {sh }} \simeq 10^{2} \mathrm{~W}$. This means that successful 
operation of the X-ray HGHG FEL requires a seed power of about $100 \mathrm{GW}$, but this value is beyond the output power of the FEL amplifier at saturation $\left(P_{\text {sat }} \simeq 10 \mathrm{GW}\right)$. In principle, in this situation a laser pulse of $100 \mathrm{GW}$ power level and a first undulator with a few periods only could be used. However, this does not solve the problem of the noise degradation, because the present analysis is based on assuming an ideal frequency multiplier chain. Considering the other contributions to the noise output, it is obvious that the $100 \mathrm{GW}$ level power is not attainable in the radiator undulator operating at the second (or higher) harmonic. This means that the condition $\left(P_{\mathrm{s}} / P_{\mathrm{n}}\right)_{\text {out }} \gg 1$ will be violated when taking into account of the second HGHG stage contribution to the noise output.

Recently, various HGHG schemes have been proposed to improve the performance of X-ray FEL. The basic theory of these schemes does not take into account the shot noise effect, meanwhile it leads to a dramatic degradation of the quality of the output radiation when applying frequency multiplication schemes. The arguments discussed above, based on our results of numerical simulations, seem to be strong enough to suggest that the HGHG FEL schemes for reaching hard X-rays proposed in the literature so far will not work.

\section{Acknowledgments}

We thank J.R. Schneider and D. Trines for interest in this work and support. 


\section{References}

[1] "TESLA Technical Design Report" (Editors: F. Richard, J. R. Schneider, D. Trines, A. Wagner)TESLA Report 2001-23, TESLA-FEL Report 2001-05, Hamburg, 2001

[2] "Linac Coherent Light Source (LCLS) Design Study Report", SLAC Report No. SLAC-R521,1998

[3] J. Feldhaus et al., Optics Communications 140(1997)341

[4] E. L. Saldin, E. A. Schneidmiller and M. V. Yurkov, Nucl. Instrum. and Methods A $445(2000) 178$

[5] "Seeding Option for the VUV Free Electron Laser at DESY": Proposal. Available at DESY by request only

[6] R. Bonifacio, L. De Salvo, and P. Pierini, Nucl. Instrum. and Methods A293(1990)627

[7] L. H. Yu, Phys. Rev. A44(1991)5178

[8] I. Ben-Zvi et al., Nucl. Instrum. and Methods A304(1991)151

[9] I. Ben-Zvi et al., Nucl. Instrum. and Methods A393(1997)II-10

[10] L. H. Yu and I. Ben-Zvi, Nucl. Instrum. and Methods A393(1997)96

[11] L. H. Yu et al., Nucl. Instrum. and Methods A445(2000)301

[12] A. Doyuran et al., "Characterization of a High-Gain Harmonic- Generation Free Electron Laser at Saturation" BNL-68031, submitted to Phys. Rev. Lett. January 2001

[13] L. H. Yu "Seeded FELs and Expected performance", Proceedings of the ICFA Advansed Beam Dynamics Workshop on Future Light Sources, C. E. Eyberger, Ed., Argone National Laboratory, Argonne, IL (1999).

[14] J. Rossbach, Nucl. Instrum. and Methods A 375(1996)269

[15] "Development of a pump-probe facility with sub-picosecond time resolution combining a high-power optical laser and a soft X-ray free electron laser": Proposal. Available at DESY by request only

[16] A. Faltens and L. J. Laslett, Part. Accel. 4(1973)151

[17] J. B. Murphy, S. Krinsky and R. L. Gluckstern, Part. Accel. 57(1997)9

[18] E. L. Saldin, E. A. Schneidmiller and M. V. Yurkov, "The physics of Free Electron Lasers" Springer-Verlag, Berlin, 1999

[19] E. L. Saldin, E. A. Schneidmiller and M. V. Yurkov, Nucl. Instrum. and Methods A $429(1999) 233$

[20] W. P. Robins "Phase noise in signal sources" IEE Telecommunications series 9, Peter Peregrinus Ltd, 1982 